ARTICLE

\title{
SVIP is a molecular determinant of lysosomal dynamic stability, neurodegeneration and lifespan
}

\author{
Alyssa E. Johnson 1,5, Brian O. Orr ${ }^{1}$, Richard D. Fetter (10 1, Armen J. Moughamian 1,2, Logan A. Primeaux ${ }^{3}$,

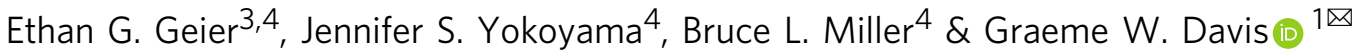

Missense mutations in Valosin-Containing Protein (VCP) are linked to diverse degenerative diseases including IBMPFD, amyotrophic lateral sclerosis (ALS), muscular dystrophy and Parkinson's disease. Here, we characterize a VCP-binding co-factor (SVIP) that specifically recruits VCP to lysosomes. SVIP is essential for lysosomal dynamic stability and autophagosomal-lysosomal fusion. SVIP mutations cause muscle wasting and neuromuscular degeneration while muscle-specific SVIP over-expression increases lysosomal abundance and is sufficient to extend lifespan in a context, stress-dependent manner. We also establish multiple links between SVIP and VCP-dependent disease in our Drosophila model system. A biochemical screen identifies a disease-causing VCP mutation that prevents SVIP binding. Conversely, over-expression of an SVIP mutation that prevents VCP binding is deleterious. Finally, we identify a human SVIP mutation and confirm the pathogenicity of this mutation in our Drosophila model. We propose a model for VCP disease based on the differential, cofactor-dependent recruitment of VCP to intracellular organelles.

\footnotetext{
${ }^{1}$ Department of Biochemistry and Biophysics, Kavli Institute for Fundamental Neuroscience, University of California, San Francisco, San Francisco, CA 94158 USA. ${ }^{2}$ Department of Neurology, University of California, San Francisco, San Francisco, CA 94158, USA. ${ }^{3}$ Department of Biological Sciences, Louisiana State University, Baton Rouge, LA 70803, USA. ${ }^{4}$ Department of Neurology, Memory and Aging Center, University of California, San Francisco, San Francisco, CA 94158, USA. ${ }^{5}$ Present address: Department of Biological Sciences, Louisiana State University, Baton Rouge, LA 70803, USA. ${ }^{凶}$ email: Graeme.davis@ucsf.edu
} 
$\mathrm{T}$ he Valosin-Containing Protein (VCP) gene encodes an $\mathrm{AAA}+\mathrm{ATPase}^{1}$ that has been linked to a broad spectrum of autosomal dominant degenerative diseases including IBMPFD (inclusion body myopathy with Paget's disease and fronto-temporal dementia; Watts et al. ${ }^{2}$ ), amyotrophic lateral sclerosis (ALS) ${ }^{3,4}$, spastic paraplegia ${ }^{5}$, muscular dystrophy ${ }^{6}$, and Parkinson's disease ${ }^{7,8}$. Patients with IBMPFD generally present to the clinic with muscle weakness in mid to late life ${ }^{4,9,10}$. Approximately one-third of patients have degeneration of the central nervous system including the fronto-temporal lobes ${ }^{4,9}$.

The $V C P$ gene is cell lethal when deleted ${ }^{11,12}$. As a likely consequence, disease-causing $V C P$ mutations are primarily distributed in the $\mathrm{N}$-terminal regulatory region, a substrate/co-factor binding domain. To date, despite a wealth of information regarding VCP activity, there is no clear consensus regarding how mutations in the VCP co-factor binding domain cause disease pathogenesis. For example, there is considerable debate regarding whether disease-causing mutations are gain-of-function versus loss-of-function mutations $7,13-16$. Furthermore, although defects in protein clearance mechanisms appear to be a root cause of $V C P$-related disease ${ }^{17}$, consistent with the general characterization of VCP as a ubiquitin-dependent protein segregase ${ }^{18,19}$, there remains debate regarding which protein clearance pathways are compromised given that VCP participates in ER-associated degradation $(\mathrm{ERAD})^{20}$, autophagy ${ }^{15,16}$, mitophagy ${ }^{7,21}$, and ubiquitin-proteasome-associated degradation (UPS) ${ }^{18}$. Although pathogenic mutations in VCP do not seem to impair the UPS or ERAD protein degradation pathways ${ }^{13,14}$, impaired autophagy ${ }^{14}$, mitochondrial quality control ${ }^{22,23}$, and lysosomal dysfunction ${ }^{16}$ have been proposed as cell biological consequences of diseasecausing VCP mutations.

One possibility is that VCP is an essential enzymatic resource that is dynamically deployed to different organelle systems, as necessary, to resolve sites of local cellular stress (Fig. 1a). In this model, the critical determinants of cellular robustness are the cofactors that bind VCP and dictate the distribution of VCP throughout the cell. According to this model, disease-causing mutations in the co-factor binding domain could, conceivably, result in both gain and loss-of-function effects if a fixed amount of VCP enzyme becomes errantly redistributed within the cell in the absence of binding to a specific co-factor. These effects might be exacerbated under conditions of cellular stress and accelerate during disease progression. With this model in mind, we set out to identify and characterize the function of VCP-binding proteins, focusing on currently unknown mechanisms that localize VCP to the lysosome.

We previously identified an essential activity for VCP at Drosophila muscle lysosomes ${ }^{16}$. We demonstrated that muscle lysosomes form a dynamic, tubular lattice that extends throughout individual muscle cells (insect and mammalian muscle). When VCP is mutated, or when VCP is acutely depleted from the cytosol, tubular lysosomes fragment, and autophagosome-lysosome fusion is blocked, ultimately leading to impaired autophagy, impaired protein clearance, and mitochondrial defects ${ }^{16}$. Fragmentation of muscle lysosomes is one of the earliest cellular defects known to occur following the disruption of $\mathrm{VCP}^{16}$. Notably, patients with disease-causing mutations in $V C P$ present to the clinic with primary muscle weakness ${ }^{10}$, highlighting the potential relevance of VCPdependent control of muscle lysosomes and autophagy. We sought to identify co-factor(s) that bind and recruit VCP to the newly identified, highly dynamic system of muscle tubular lysosomes.

In this study, we define the function of a VCP-interacting cofactor termed SVIP (Small VCP-Interacting Protein) ${ }^{24,25}$. SVIP was initially identified as a VCP-interacting protein and a negative regulator of ERAD ${ }^{24,25}$. Prior work in immortalized human cells (HeLa) demonstrated that SVIP over-expression is sufficient to alter the subcellular distribution of VCP to "perinuclear granules" and the plasma membrane ${ }^{26}$. SVIP knockdown correlated with altered LC3 lipidation in these cells. However, beyond this, little is known about SVIP function.

Here we define SVIP as an essential VCP co-factor that recruits VCP to lysosomes, an activity that is essential for the dynamic stability of the muscle tubular-lysosomal lattice. Loss of SVIP disrupted the muscle lysosomal network, impaired autophagosome-lysosome fusion, and caused myriad degenerative defects including muscle wasting, neuromuscular degeneration, dendrite degeneration in motoneurons, progressive motor dysfunction and decreased organismal lifespan. We link SVIP to VCP-dependent degenerative disease through phenotypic analysis of a known human disease-causing mutation in Drosophila VCP, and we present the identification of an SVIP mutation in a human patient diagnosed with fronto-temporal dementia (FTD). We subsequently confirmed the pathogenicity of this human SVIP mutation in our Drosophila model. Although we cannot conclude causality, this human mutation and our subsequent characterization in vivo in Drosophila argues that the function of SVIP has relevance to the human condition. Taken together, our data define a model for VCP disease based on the differential, co-factor-dependent recruitment of VCP to intracellular organelles. Further, our data underscore the relevance of lysosome dysfunction as an underlying cause for VCP-linked diseases of the peripheral and central nervous systems.

\section{Results}

Drosophila SVIP recruits VCP to tubular lysosomes. We identified a small ORF (CG32039) that shares high sequence homology to the human, mouse, and rat SVIP proteins (Fig. 1b). Critical residues are conserved in CG32039 including glycine 2 that is required for SVIP myristoylation and membrane tethering, as well as the region encompassing a putative VCP-interaction motif (VIM) (Fig. 1b). Thus, based on sequence homology, CG32039 (hereafter referred to as SVIP) is a strong candidate to be the Drosophila SVIP ortholog.

We first demonstrate that Drosophila SVIP directly binds to VCP. Purified recombinant GST-SVIP and MBP-VCPN-D1 (containing the $\mathrm{N}$-terminal and first D1 ATPase domain) proteins directly bind and this interaction was lost when two arginine residues $(\mathrm{R} 24,25)$ in the VIM sequence of SVIP $\left(\mathrm{SVIP}^{\mathrm{RR}}\right)$ were mutated to alanine (Fig. 1c). Additionally, mutation of VCP aspartate 32, a residue previously demonstrated to disrupt VCP interaction with VIM motifs ${ }^{27}$ also disrupted the SVIP-VCP interaction in vitro (Supplementary Fig. S1A, B). Thus, we define SVIP as a VCP-binding protein.

In order to define the subcellular distribution of SVIP, we took two approaches. First, we generated an antibody against GSTSVIP to examine the endogenous expression patterns of Drosophila SVIP. SVIP is expressed in both 3rd instar larvae and adults, being expressed at relatively lower levels in the brain compared to the rest of the body (Supplementary Fig. S1C). Second, we used CRISPR to knock-in a superfold GFP (sfGFP) tag at the $\mathrm{C}$ terminus of the endogenous SVIP gene (see "Methods" section). This approach allows us to assess SVIP localization in live tissue. We demonstrate that SVIP is expressed in the body wall muscles of 3rd instar larvae where it co-localizes with a lysosomal marker Spin-RFP (Fig. 1d, e).

We previously demonstrated that muscle lysosomes form a dynamic tubular lattice that extends throughout the muscle cytoplasm $^{16}$. This dynamic lattice can be observed by imaging an RFP tagged integral lysosomal membrane protein (Spin-RFP; 
a

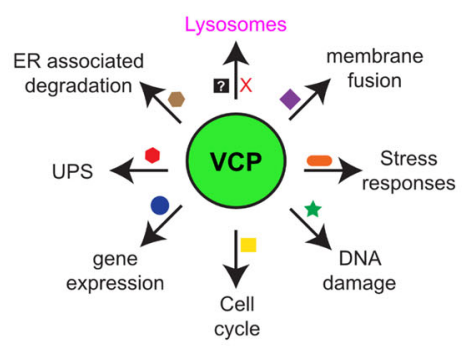

b

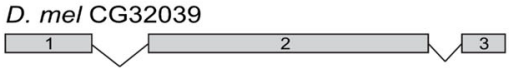

C

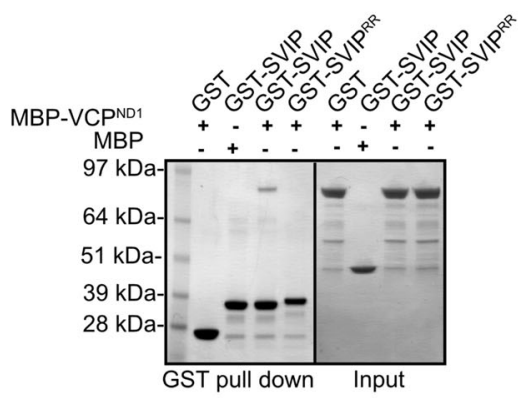

d
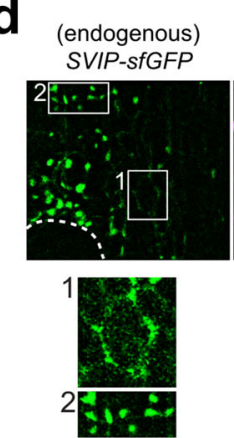
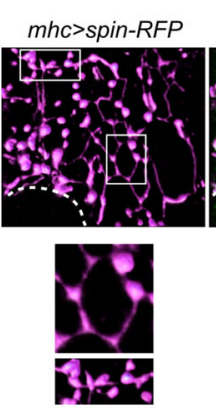

$1 \square$

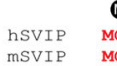

MSVIP
rSVIP

CG32039

histe

MSVIP

CG32039

hSVIP

mSVIP

CG32039
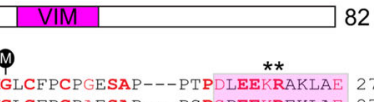

GLCFPCPAESAP---PSPS PEEKREKLAE MGLCFPCPAESAP---PSPSPEEKRAKLAE 27

AERROKSATSRGIIDVOSVQEKRKKKEKI 57 AERRQKEAATRGILDIQSVEAKKKKKEQL 5 AAEKRRQENEHRGIKNPDSVRROQORAEM

RQIA--TSGPPPEGGLRWTVS 77 KQMA--TSGPPTAGGLRWTVS 77

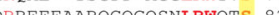

\section{e \\ f}

merge

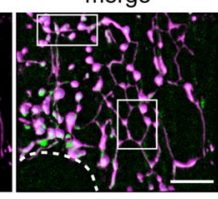

g

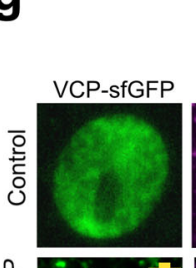

$\underline{\text { nuclear }}$
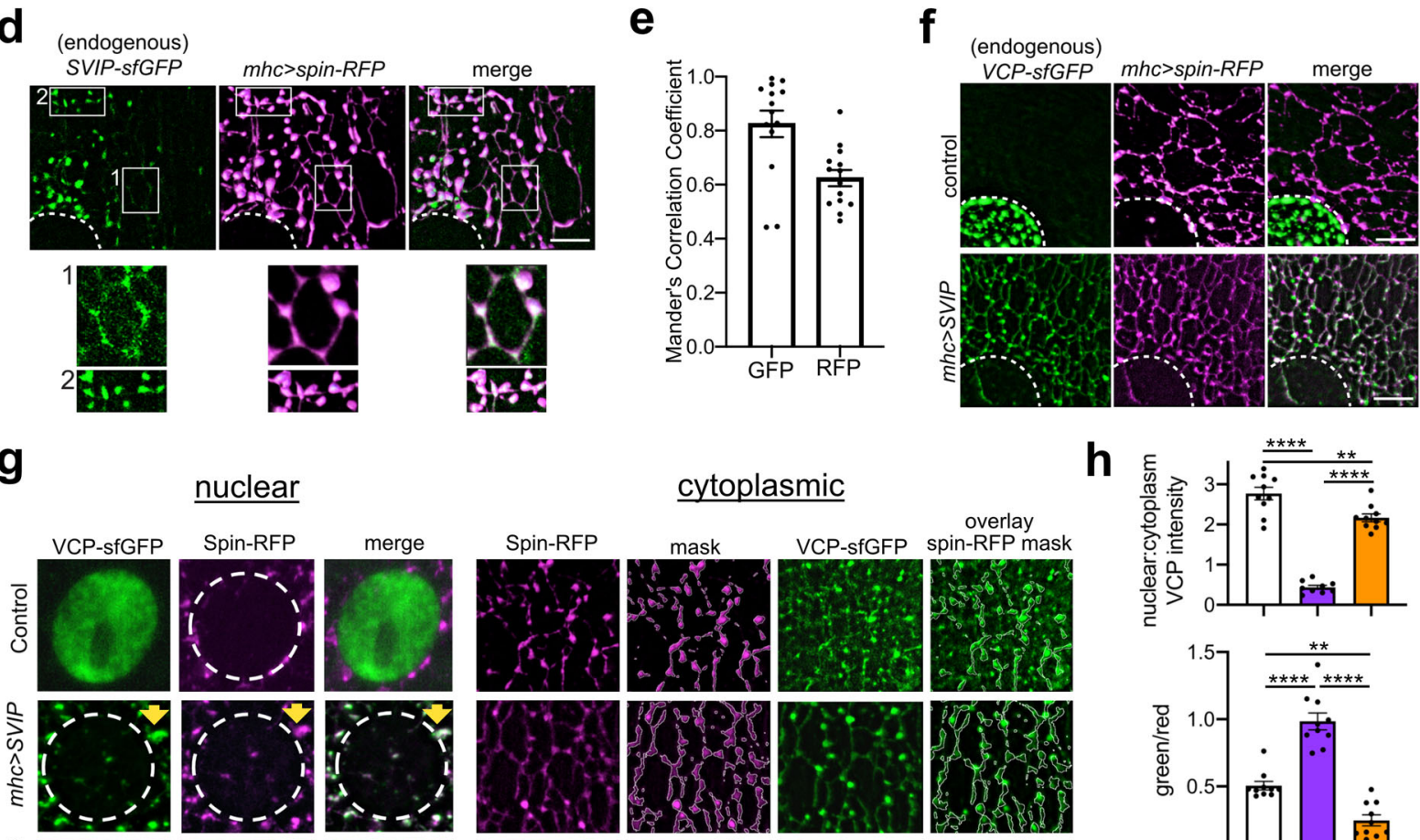

merge

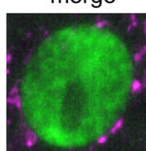

Spin-RFP

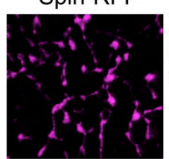

cytoplasmic
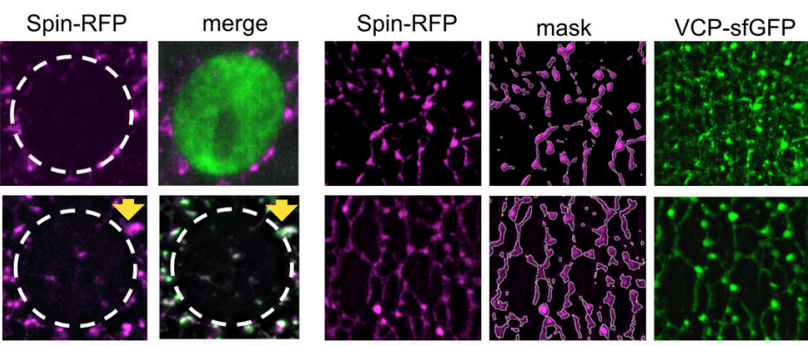

overlay
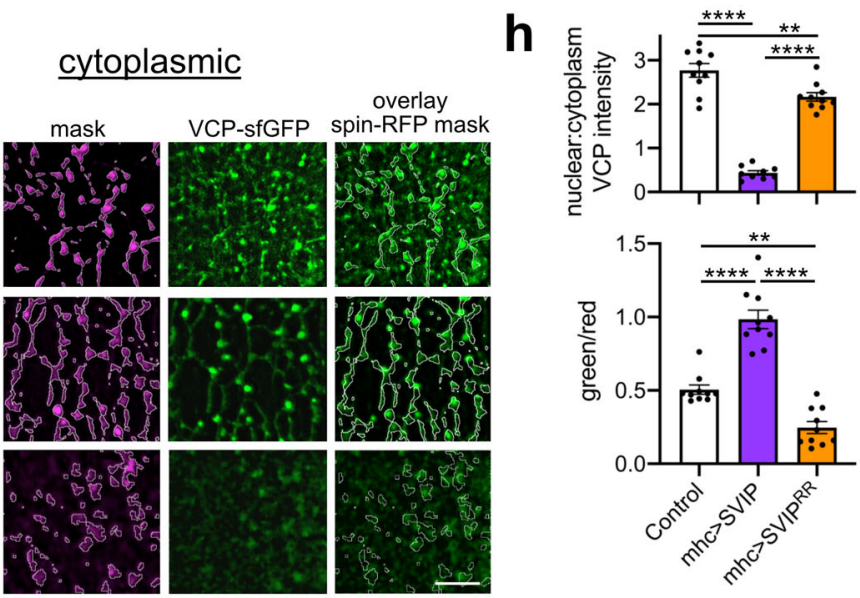

Fig. 1 Drosophila SVIP directly recruits VCP to tubular lysosomes in muscles. a Distinct co-factors (shapes of different colors adjacent to arrows) spatially regulate VCP subcellular distribution and function. b Schematic of Drosophila CG32039 gene and protein organization (top) and sequence alignment with human, mouse, and rat SVIP orthologs (bottom). The region shaded in magenta corresponds to the predicted VCP-interaction motif (VIM), $M$ indicates myristoylation site, asterisks indicate arginine residue mutated in part $C$, and serine highlighted in yellow indicates SVIP patient mutation identified (Fig. 9). c In vitro binding of recombinant MBP-VCP and GST-SVIP fusion proteins. d Co-localization of endogenously tagged SVIP-sfGFP and Spin-RFP (lysosomes) in Drosophila muscles. The dotted line indicates the nucleus. Boxes indicated insets, below at higher magnification. e Mander's correlation coefficients for SVIP-sfGFP (GFP) and Spin-RFP (RFP) signal ( $n=13$ muscle cells examined over five independent animals). f Co-imaging of VCP-sfGFP and Spin-RFP in control and SVIP+ over-expression in muscles. Dotted lines as in $\mathbf{d}$. $\mathbf{g}$ Co-imaging of endogenously tagged VCP-sfGFP and UASSpin-RFP (lysosomes) in control compared to SVIP+ and SVIPRR over-expression in muscles. Dotted lines as in $\mathbf{d}$ and $\mathbf{f}$. Yellow arrow (middle row) highlights signal co-localization. h Nuclear to cytoplasmic ratios of VCP-sfGFP signal was quantified for the genotypes indicated (top graph). Spin-RFP signal was used to create a masked ROI (white lines, "mask") and plots represent GFP/RFP signal intensities within the masked region (bottom graph, see "overlay" in $\mathbf{g}$ ). Data, artificial imaging units. ( $n=10$ muscle cells examined over five independent animals). See also Supplementary Fig. S1. Scale bars $5 \mu \mathrm{m}$. Data presented as mean and SEM. Student's $t$-test for individual comparisons $\left({ }^{\star} p<0.05 ;{ }^{\star \star} p<0.01 ;{ }^{\star \star \star} p<0.001,{ }^{\star \star \star \star} p<0.0001\right)$.

Supplementary Movie 1). This tubular-lysosomal system is present in a diversity of cells and organisms, inclusive of cultured mouse myoblasts (ref. ${ }^{16}$ and unpublished observation). Importantly tubular lysosomes are only visible in live tissue, collapsing into vesicular structures by a diverse array of fixation methods ${ }^{16}$. Here we demonstrate that SVIP localizes at lysosome tubules that are distributed throughout the cytosol (see Fig. 1d, insets 1 and 2 as well as quantification in Fig. 1e).
To determine whether SVIP recruits VCP to muscle tubular lysosomes, we tested whether over-expressing Drosophila SVIP drives enhanced VCP localization to lysosomes. Because muscle lysosomes do not retain their dynamic tubular structure following fixation, we generated two transgenic animals to allow us to overexpress SVIP and monitor endogenously tagged VCP protein in vivo. First, we created a UAS-SVIP transgenic animal enabling SVIP over-expression. Second, we used CRISPR technology to tag 
VCP with sfGFP at its endogenous locus. Using these tools, we demonstrate that endogenous VCP protein concentrates on the nuclear membranes in muscle and is weakly distributed throughout the rest of the muscle cell. Image analysis of extranuclear VCP reveals a significant concentration at tubular lysosomes (Fig. 1f, g; note that exposure time for VCP-sfGFP is extended in ( $g$ ) compared to ( $f$ ) to enable visualization of lysosome-associated VCP). Strikingly, when UAS-SVIP is overexpressed using the muscle-specific $m h c-G A L 4$ driver, endogenously expressed VCP-sfGFP re-localizes to tubular lysosomes throughout the muscle cell (Fig. 1f, g). The SVIP-dependent relocalization of VCP causes depletion of VCP from the perinuclear compartment where VCP is enriched at a steady state (Fig. 1f, g). As a control, we over-expressed UAS-SVIPRR, an SVIP variant that cannot bind VCP (Fig. 1c). As expected, $S V I P^{R R}$ over-expression did not induce VCP re-localization to the tubular-lysosomal network (Fig. 1f, g). We quantified these effects by measuring two parameters. First, we quantified the juxta-nuclear to cytoplasmic (representative of lysosomal tubules located throughout cytoplasm) ratio of endogenously tagged VCP-sfGFP (Fig. 1h). Second, we used the Spin-RFP channel to create a mask of the tubular-lysosomal network and quantified VCP-sfGFP intensity in this region of interest (Fig. 1g, h). Collectively, these data demonstrate that Drosophila SVIP binds VCP and is sufficient to re-localize endogenous VCP to the tubular-lysosomal system.

SVIP-dependent VCP recruitment is essential to stabilize lysosomal structure and function. To investigate the function of SVIP in vivo, we generated an SVIP mutant using the CRISPR-Cas9 system. We designed a gRNA to direct Cas9 to cut within the first exon of SVIP, which resulted in a frame-shift mutation and a pre-mature stop codon upstream of the VIM site (Fig. 2a). Using our SVIP antibody, we validated that the SVIP mutation is a protein null by western analysis (Fig. 2a; hereafter referred to as $S V I P^{K O}$ ).

We previously demonstrated that VCP mutations cause fragmentation of the tubular lysosome system in Drosophila muscle ${ }^{16}$. If SVIP is necessary to recruit VCP to lysosomes, then the loss of SVIP should phenocopy the VCP mutant. Prior live imaging studies demonstrate that lysosome tubules are highly dynamic with constant tubule extension, scission, and fusion throughout the muscle cytoplasm ${ }^{16}$. First, we repeat this live imaging (Supplementary Movie 1). Next, we demonstrate that the SVIPKO causes fragmentation of the network of lysosome tubules in body wall muscles of 3rd instar larvae (Fig. 2b). Two measures were used to quantify effects on the lysosomal tubular network: (1) the number of junctions per tubule, which assesses the complexity of the tubule network, and (2) the number of tubules within a $100 \mu \mathrm{m}^{2}$ region of interest, as a measure of tubule density. Both measures are significantly decreased in the SVIPKO (Fig. 2b, pink). This effect requires the SVIP-VCP interaction because over-expression of wild-type UAS-SVIP in the SVIPKO fully rescues the tubular lysosome system (Fig. 2b, rescue, green), whereas over-expression of the UAS-SVIPRR mutant that abolishes SVIP-VCP binding does not rescue (Fig. 2b, SVIPRR, orange). Finally, we note that over-expression of UAS-SVIP not only restores the lysosomal network but increased tubule density beyond wild-type levels $(p<0.001)$, suggesting that SVIP levels may be limiting for the dynamic stability of the lysosomal tubule network.

We also extend these observations to the adult Drosophila abdominal muscles, demonstrating that the SVIPKO disrupts tubular-lysosomal networks (Fig. 2c). We demonstrate a significant drop in tubule complexity (junctions per tubule), but there was only a trend toward fewer tubules. On closer inspection of the collapsed lysosomal network, we find that tubules can still emanate from vesicular lysosomes (Fig. 2c, white arrows). Thus, in the absence of SVIP-dependent recruitment of VCP, it appears that lysosomes retain the ability to generate de novo tubules at a basal rate, but tubules appear unable to fuse with other lysosomes and cannot sustain a stable lattice.

We previously demonstrated that VCP is required for the fusion of autophagosomes with lysosomes, a process that sustains the integrity of the tubular-lysosomal system ${ }^{16}$. To test the involvement of SVIP in VCP-mediated autophagosome-lysosome fusion, we used a dual fluorescent autophagy sensor: mCherryGFP-Atg8a. Atg8a resides in the membrane of mature autophagosomes. When autophagosomes fuse with lysosomes, GFP fluorescence is quenched by low lysosomal $\mathrm{pH}$, but the mCherry signal is unaffected. Therefore, the efficiency of autophagosome-lysosome fusion can be measured by the amount of GFP fluorescence compared to the amount of mCherry signal. Here, we demonstrate that $S V I P^{K O}$ animals display significantly increased GFP fluorescence compared to wild type (Fig. $2 \mathrm{~d} ; p<$ 0.001). Thus, the ability of autophagosomes to fuse with lysosomes is defective. Consistent with such a defect, SVIPKO animals also accumulated detergent-insoluble protein aggregates (Supplementary Fig. S2). Collectively, these data argue that SVIPmediated VCP recruitment to muscle lysosomes promotes both homotypic lysosome fusion (to sustain the tubular-lysosomal lattice) and heterotypic membrane fusion with autophagosomes (Fig. 2d).

Synthetic reconstitution of VCP-lysosomal targeting rescues lysosomes in $S V I P^{K O}$. To further address our emerging model, we asked whether synthetically driving VCP to lysosomes in the SVIPKO is sufficient to rescue the tubular lysosome lattice. To do so we designed a system based on FKBP-FRB (FK506 Binding Protein-FRB) dimerization to artificially drive VCP to lysosomes. A variant of FKBP $\left(\mathrm{FKBP}^{\mathrm{F} 37 \mathrm{~V}}\right)$ that binds to the rapamycin analog $\mathrm{B} / \mathrm{B}$ dimerizer was fused to the $\mathrm{N}$ terminus of the lysosome-specific membrane protein Spinster ${ }^{16,28}$. The complementing FRB tag was fused to the $\mathrm{N}$ terminus of VCP (Fig. 2e). To visualize the two proteins, mCherry and Venus tags were fused to the $\mathrm{C}$ terminus of Spinster and VCP, respectively (Fig. 2e). In $S V I P^{K O}$ animals, in the absence of the $\mathrm{B} / \mathrm{B}$ dimerizer, lysosomes were vesicular and VCP appeared diffuse throughout the cytoplasm (Fig. 2f, top panels, control). However, after $4 \mathrm{~h}$ of $\mathrm{B} / \mathrm{B}$ incubation, VCP re-localized to lysosomes, and the tubularlysosomal lattice began regenerating (Fig. 2f, bottom panels, dimerizer). We find that the increase in tubule density in the presence of dimerizer is highly significant $(p<0.001)$, and there is a strong trend toward increased tubule complexity $(p=0.09)$. Thus, synthetic recruitment of VCP to lysosomes, performed in the absence of SVIP which normally localizes VCP to lysosomes, is sufficient to enhance the stability of a dynamic lysosomal network. The inability to fully generate a network, inclusive of significant increases in the number of junctions per tubule could be due to the short time $(4 \mathrm{~h})$ of dimerizer or reflect a slight impairment of reconstituted VCP activity due to the engineered dimerizer system.

SVIP functions in muscle, affecting organismal motility, and lifespan. We sought to assess the organismal effects of SVIPKO and define which tissues require SVIP function. In the 3rd instar larvae, we observed no apparent impairment of motility (Supplementary Fig. S3A) and no hallmarks of motor neuron or muscle degeneration (Supplementary Fig. S3B). However, we found that $28 \%$ of $S V I P^{K O}$ pupa failed to eclose to the adult stage, 


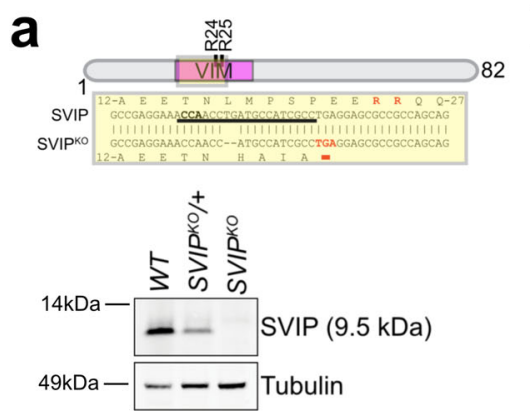

b
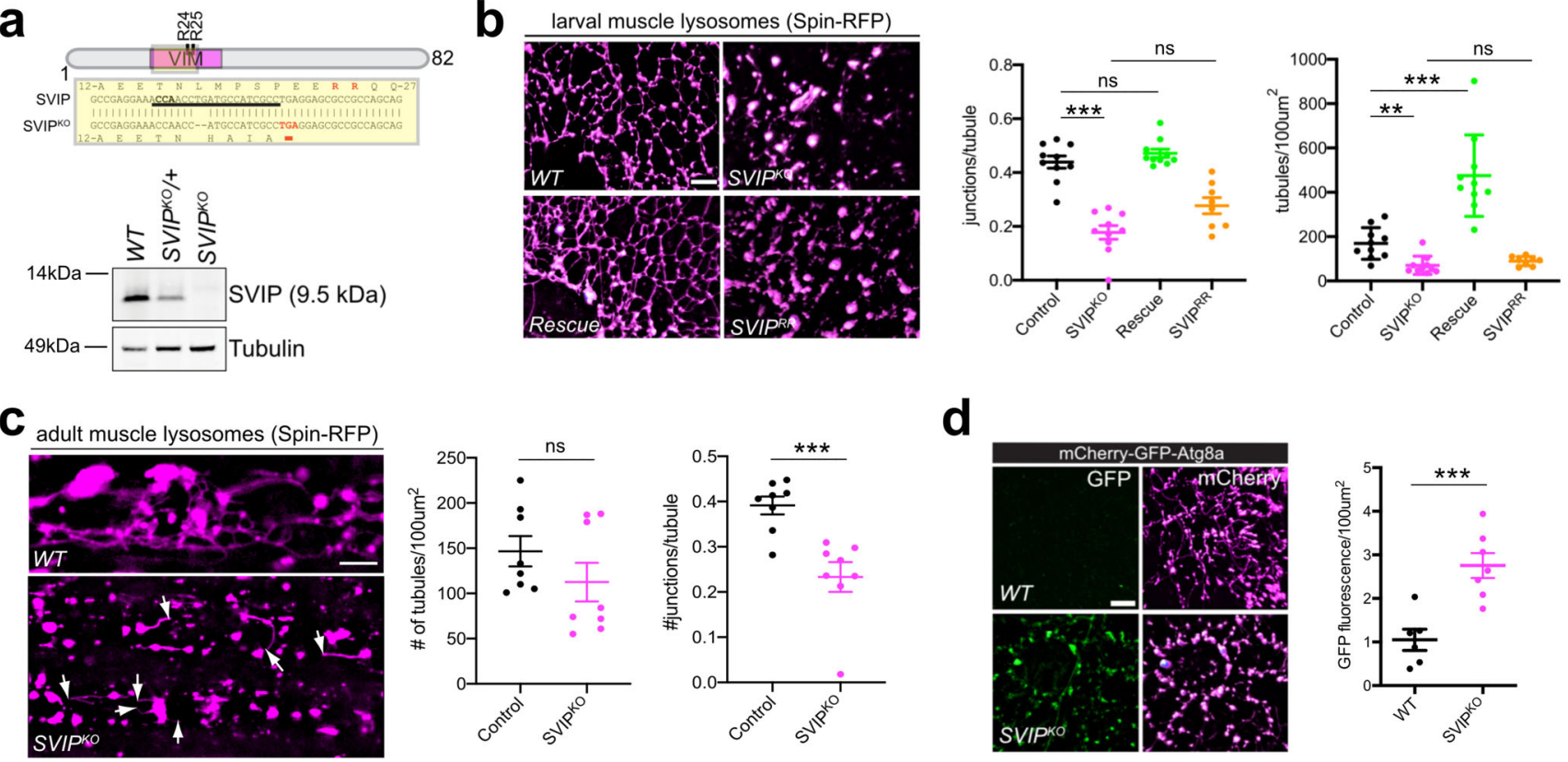

e

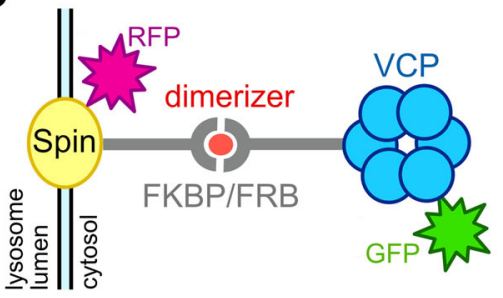

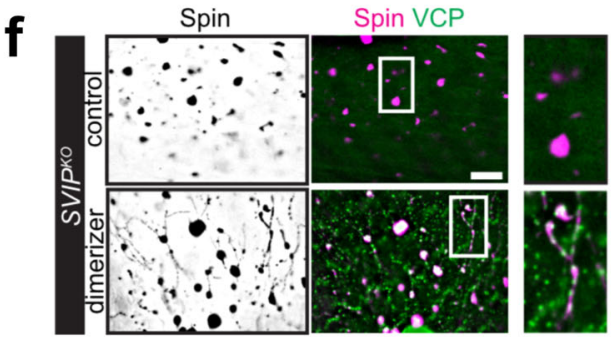
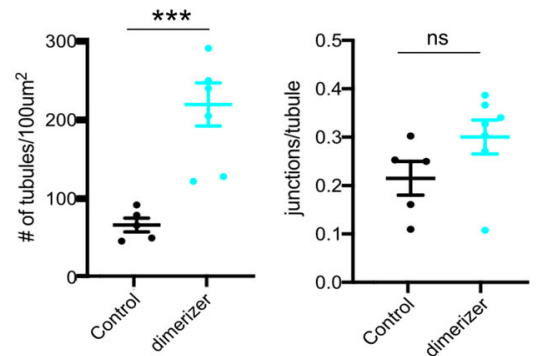

Fig. 2 SVIP-dependent VCP recruitment to lysosomes is required for lysosome integrity and function. a Schematic of SVIPKO mutant generated by CRISPR (top) and western blot analysis of SVIP protein levels in wild type (WT), SVIPKO heterozygous and SVIPKO homozygous mutants (bottom). b Representative images of Spin-RFP in 3rd instar larval muscles for the indicated genotypes. Quantification (right) for indicated genotype for tubule complexity (junctions per tubule) and tubule density (tubules $\left./ 100 \mu \mathrm{m}^{2}\right) .(n=10$ muscle cells examined over five independent animals). c Representative images of Spin-RFP in 1-week-old adult abdominal muscles for the indicated genotypes. Quantification as in $\mathbf{b}$. ( $n=8$ muscle cells examined over four independent animals). d Representative images of mCherry-GFP-Atg8a (autophagy sensor) in wild type (WT) and SVIPKO larval muscles. Quantification at right. ( $n=7$ muscle cells examined over three independent animals). e Cartoon diagram of FKBP-FRP dimerization system (components as indicated). f Representative images of FKBP-Spin-mCherry and FRB-VCP-Venus localization in the absence (control) or presence of B/B dimerizer. Quantification at right as in $\mathbf{b}$. ( $n=6$ muscle cells examined over three independent animals). Scale bars, $5 \mu \mathrm{m}$. See also Supplementary Fig. S2. Data presented as mean and SEM. Student's $t$-test for individual comparisons $\left({ }^{\star} p<0.05 ;{ }^{\star \star} p<0.01 ;{ }^{\star \star \star} p<0.001\right)$. ANOVA for multiple comparisons $\left({ }^{\star} p<0.05 ;{ }^{\star \star} p<0.01 ;{ }^{* \star \star} p<0.001\right)$.

compared to just $7 \%$ of wild-type animals (Supplementary Fig. S3C). These data argue that SVIP function is relevant to organismal development and may be relevant to health and lifespan. Thus, we turned to the analysis of adult animals where these parameters can be addressed.

In adult Drosophila, we find that SVIPKO animals have a reduced lifespan compared to controls $(29 \%$ decrease in median lifespan) (Fig. 3a). We next asked where SVIP is required for a normal lifespan. Remarkably, diminished lifespan can be fully rescued by muscle-specific expression of UAS-SVIP (Fig. 3a). By contrast, neuronal expression of UAS-SVIP in the SVIPKO did not rescue viability (Supplementary Fig. S4A). Since SVIP is necessary and sufficient in skeletal muscle to control autophagosomal fusion and the dynamic stability of the lysosomal tubular network, we propose that these activities of SVIP, in muscle, are necessary for normal organismal lifespan.

Next, we asked whether the decrease in lifespan is preceded by impaired motor behavior. Motility was assessed using a commonly employed negative geotaxis assay, taking advantage of a powerful innate behavior in which animals climb the side of a vial immediately upon being knocked to the bottom. We observe a profound deficit in motor ability in SVIPKO animals that is first apparent at 14 days and progresses to a complete inability to climb the sides of a vial by 28 days (Fig. 3b). The SVIPKO at 28 days are fully viable and do not begin to show increased mortality for another 10 days (Fig. 3a). Thus, progressive motor dysfunction precedes early lethality, consistent with age-related, progressive degeneration in the motor system.

Next, we asked where SVIP is required in order to sustain normal motor behavior. To do so, we assessed climbing performance at 28 days. This time point was chosen as a moment when the $S V I P^{K O}$ is almost completely immobile, but when there remains no change in animal viability. Muscle-specific expression of UAS-SVIP in the SVIPKO restores mobility to nearly wild-type values ( $\sim 90 \%$ rescue of negative geotaxis, Fig. $3 c$ ), whereas panneuronal expression of UAS-SVIP in the SVIPKO provides only partial ( 50\%) rescue (Supplementary Fig. S4B). Thus, while there is some benefit to the expression of SVIP in the nervous system, it is apparent that wild-type animal viability and motility can be achieved when SVIP is expressed exclusively in muscle. 
a

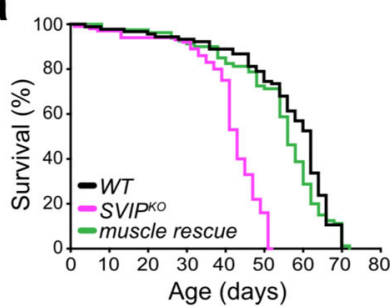

$W T(53.4), K O(42.1): p<0.001$

$W T(53.4)$, resc. $(53.3): p=0.85$

C

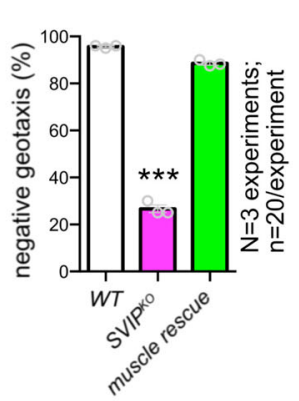

e

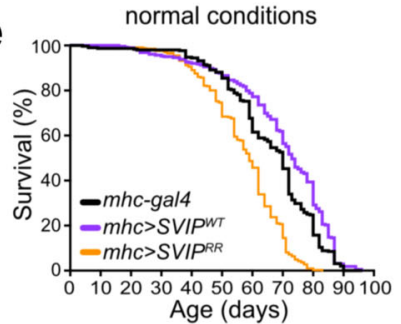

Age (days)

MHC (65.8), SVIP(wt) (69.4): $p<0.001$ MHC (65.8), SVIP(RR) (56.7) : $p<0.001$

g

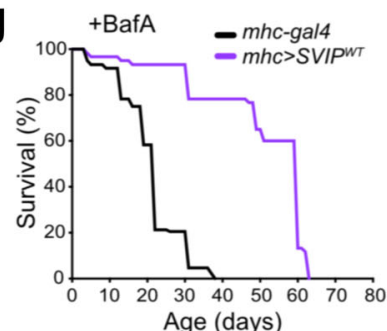

MHC (21.0), SVIP(WT) (51.8) : p<0.001

d b
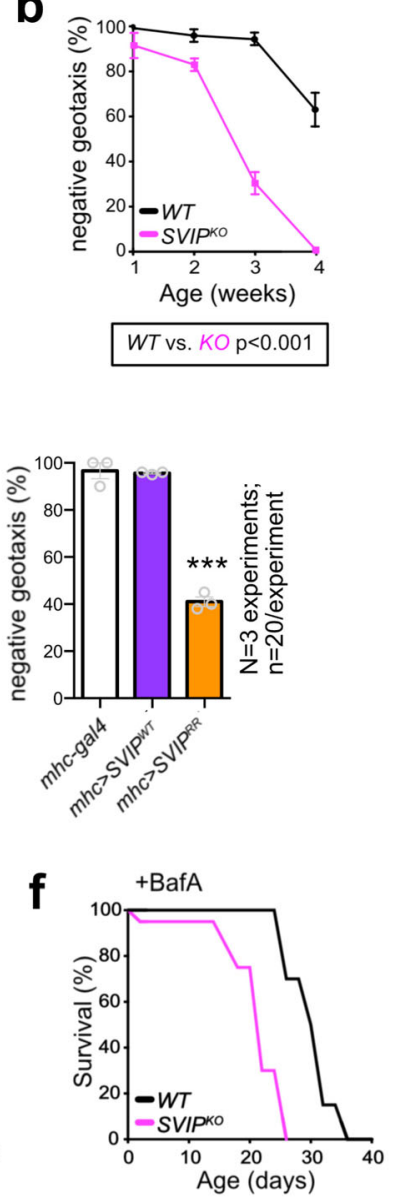

WT (30.4), KO (21.2) : $p<0.001$

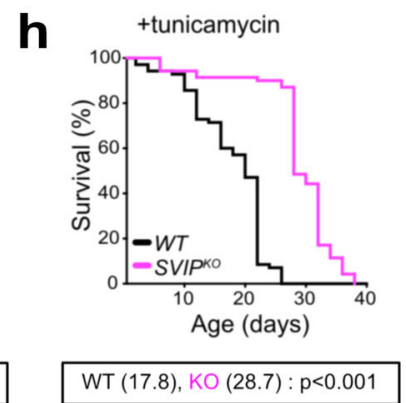

To further confirm the essential activity of muscle SVIP, and to link SVIP activity to the muscle-specific function of VCP, we compared the effects of muscle over-expression of wild-type UASSVIP versus mutant UAS-SVIPRR that is unable to bind VCP. Muscle-specific over-expression of UAS-SVIPWT was without effect, but muscle-specific over-expression of $U A S-S V I P^{R R}$ had a profound $(\sim 65 \%)$ decrease in animal mobility in our negative geotaxis assay (Fig. 3d). These data are consistent with SVIPdependent recruitment of VCP to muscle lysosomes being essential.

Next, we returned to the effects of SVIP on organismal lifespan. Given the potent activity of SVIP in muscle, we asked whether muscle over-expression of SVIP, in a wild-type background, would further extend lifespan. Indeed, lifespan extension is statistically significant when SVIP is expressed in muscle, although the absolute magnitude of the effect is rather modest

Fig. 3 SVIP is a stress-dependent survival factor in muscle. a Lifespan of $W T(n=90), \operatorname{SVIPKO}^{K}(n=100)$, and SVIPKO muscle rescue $(n=79)$. b Negative geotaxis assay for WT and SVIPKO mutants at the ages indicated. Data from three independent replicates with 20 animals each are presented as mean and SEM for each age point ( $p<0.01$ for 1 and 2 weeks, $p<0.001$ for 3 and 4 weeks). c, $\mathbf{d}$ Negative geotaxis assay at 4 weeks of age for the genotypes indicated. Data from three independent replicates with 20 animals each are presented as mean and SEM. e Lifespan of for control (mhc-gal4, $n=300$ ) and muscle over-expression of UAS-SVIPWT $(n=298)$ and UAS-SVIPRR $(n=302)$. $\mathbf{f}$ Lifespan of WT and SVIPKO mutants that were constantly fed the lysosome inhibitor, bafilomycin-A ( $n=60$ for both genotypes). $\mathbf{g}$ Lifespan quantified for control (mhc-gal4) and animals with muscle-specific over-expression of SVIPWT (mhc-gal4, UAS-SVIP) that were constantly fed the lysosome inhibitor, BafA ( $n=69$ for each genotype). $\mathbf{h}$ Lifespan of WT and SVIPKO mutants that were constantly fed the ER inhibitor, tunicamycin ( $n=70$ for each genotype). See also Supplementary Figs. S3 and S4. Mean lifespans are in parentheses. Logrank tests were used to determine statistical significance. Student's $t$-test for individual comparisons $\left({ }^{\star} p<0.05 ;{ }^{\star \star} p<0.01 ;{ }^{* \star} p<0.001\right)$. ANOVA for multiple comparisons $\left({ }^{\star} p<0.05 ;{ }^{\star \star} p<0.01 ;{ }^{\star \star \star} p<0.001\right)$.

(Fig. 3e, purple). Finally, over-expression of the SVIP mutant that is unable to bind VCP (SVIPRR), significantly diminished lifespan, as predicted (Fig. 3e, orange).

What happens when animals are stressed by the administration of drugs that disrupt the lysosome or impair normal proteostasis? Would SVIP muscle over-expression have a beneficial effect? We raised wild type and $S V I P^{K O}$ animals on food containing the lysosomal inhibitor bafilomycin-A (BafA). Under this condition, wild-type mean lifespan is decreased from $\sim 53$ days (Fig. $3 a$ ) to $\sim 30$ days (Fig. 3f), highlighting the deleterious effects of this drug delivered at concentrations previously used for feeding experiments in Drosophila ${ }^{29}$. Importantly, we find that lifespan is even more severely affected in the $S V I P^{K O}$ compared to wild type (Fig. 3f, pink), with mean lifespan diminished to $\sim 20$ days. Thus, loss of SVIP sensitizes the organism to the effects of BafA, as might be expected given that BafA targets the lysosome.

Next, we tested whether muscle-specific SVIP over-expression might counter the deleterious effects of BafA. Indeed, muscle over-expression of SVIP in wild-type animals nearly doubles lifespan in the presence of BafA (Fig. $3 \mathrm{~g} ; m h c>S V I P P^{W T}$ ). Thus, SVIP over-expression, which is sufficient to increase lysosomal density in muscle, has a beneficial effect on lifespan under conditions of lysosomal stress.

Finally, we asked whether SVIP over-expression is beneficial in the context of other pharmacological stressors. We reared animals on food containing Tunicamycin to stress the ER-resident unfolded protein response. A concentration of Tunicamycin was chosen based on prior work ${ }^{30,31}$ and which induces elevated GRP78/Bip levels in both WT and SVIPKO animals (Supplementary Fig. S4C). As predicted, wild-type mean lifespan is diminished, dropping from $\sim 53$ days (Fig. 3a) to $\sim 17$ days (Fig. $3 \mathrm{~h}$ ). As above, we repeated this assay in the SVIPKO animals. Remarkably, knockouts live dramatically longer than wild type in the presence of Tunicamycin (Fig. 3h, pink). Several possible explanations exist. Among these, SVIPKO animals could be Tunicamycin resistant because increased amounts of VCP can be deployed to the ER in the absence of SVIP-mediated localization of VCP to the lysosome. This is consistent with the initial identification of SVIP as a negative regulator of ER-associated protein degradation ${ }^{24}$. These data support our initial model in which the dynamic, co-factor-dependent deployment of VCP to different organellar systems can have powerful effects on organismal health and lifespan (Fig. 1a). 
a

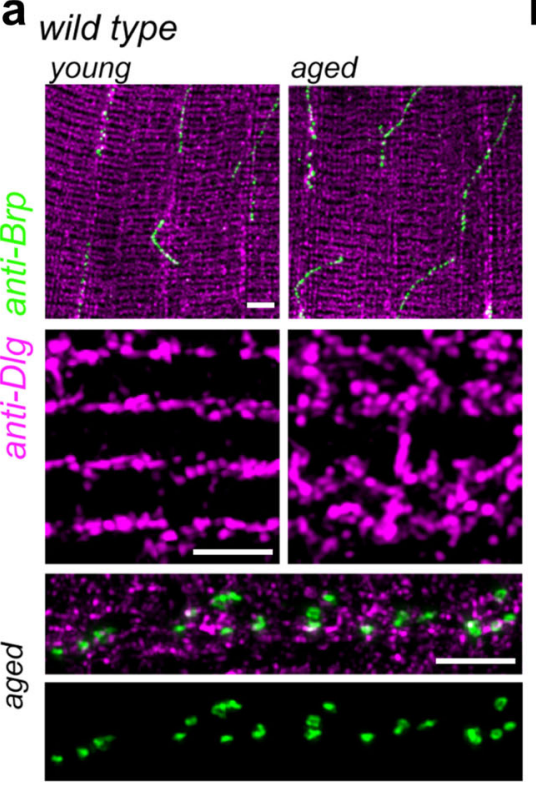

SVIP KO

young

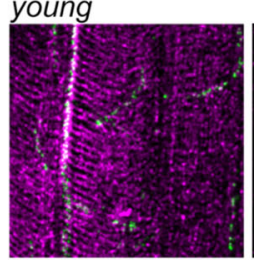

aged
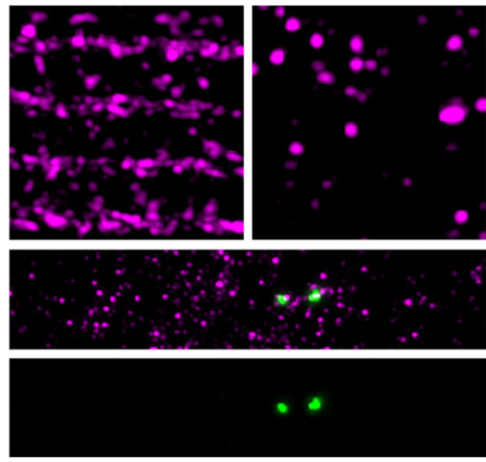

d

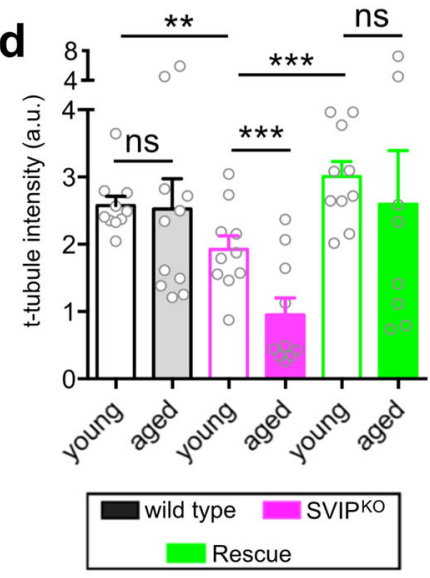

e

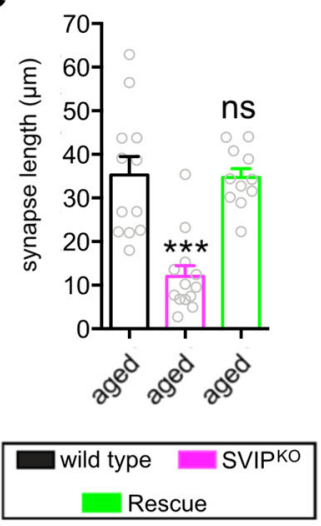

f wild type
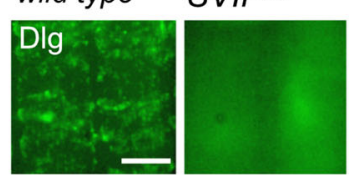

wild typ

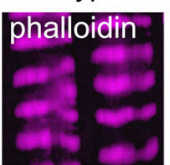

9
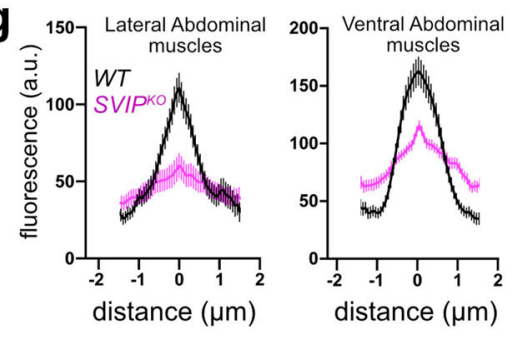

C Rescue

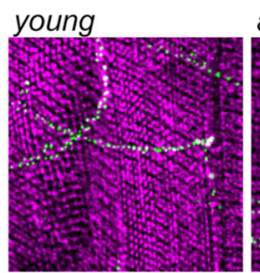
aged
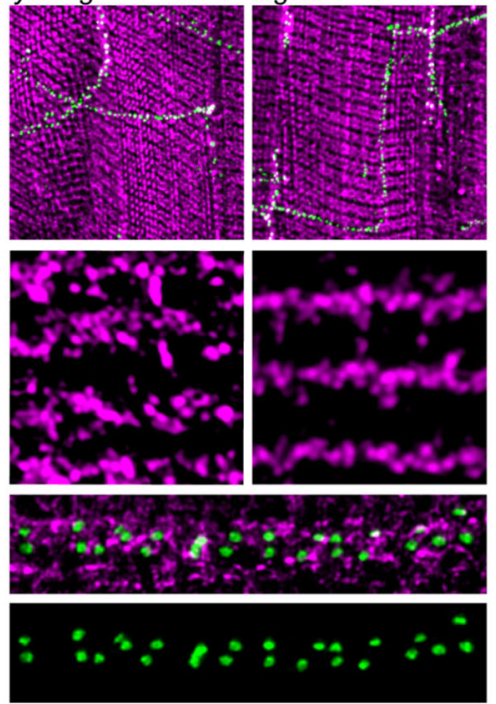

SVIPKO

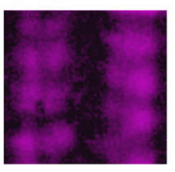

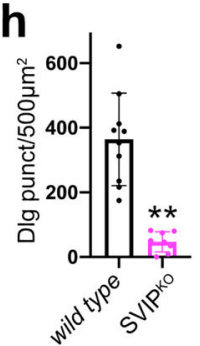

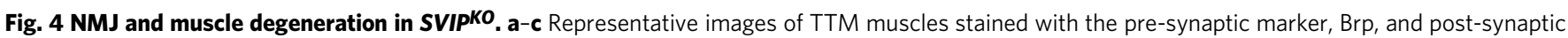
marker DLG (labels post-synaptic membranes and T-tubules). Young animals are 1-5 days post eclosion. Aged animals are 25-30 post eclosion. Insets: middle row (square boxes) show anti-DLG stained t-tubules. Bottom rows (rectangle) show the NMJ with an anti-Brp diagnostic of individual active zones. Scale bar $5 \mu \mathrm{m}$. d Quantitation of T-tubule (DLG) fluorescence intensity. (WT young $n=10$ cells examined over six independent animals, WT aged $n=$ 11 cells examined over six independent animals, SVIPKO young $n=10$ cells examined over six independent animals, SVIPKO aged $n=10$ cells examined over six independent animals, rescue young $n=10$ cells examined over six independent animals, rescue aged $n=10$ cells examined over six independent animals). e Quantitation of synapse length. (WT aged $n=12$ cells examined over six independent animals, SVIPKO aged $n=13$ cells examined over six independent animals, rescue aged $n=11$ cells examined over six independent animals). $\mathbf{f}$ Representative images of abdominal muscles stained with DLG and Phalloidin. Scale bar $5 \mu \mathrm{m}$. $\mathbf{g}$ Linescan of fluorescence intensity within single phalloidin bands (average \pm SEM for each point), data aligned to peak intensity within a band. h Quantification of DLG puncta per $500 \mu \mathrm{m}^{2}$. Data presented as mean and SEM. Student's $t$-test for individual comparisons ( ${ }^{\star} p<$ $\left.0.05 ;{ }^{\star \star} p<0.01 ;{ }^{\star \star \star} p<0.001\right)$. ANOVA for multiple comparisons $\left({ }^{\star} p<0.05 ;{ }^{\star \star} p<0.01 ;{ }^{\star \star \star} p<0.001\right)$.

Age-related muscle defects in SVIP knockout animals. IBMPFD is an age-related disorder, with patients typically presenting with muscle weakness in their fifth to sixth decade. Thus, we sought to characterize the progression of muscle wasting and neuromuscular degeneration, over time, in adult Drosophila. We assessed muscle integrity at 1 week and 4 weeks of age, comparing wild type and SVIPKO. The Dlg antibody labels the highly organized junction of T-tubule membranes with the muscle plasma membrane $^{32}$. We quantified anti-Dlg signal intensity/area (see "Methods" section). The anti-Brp antibody labels pre-synaptic Tbars that reside at sites of neurotransmitter release within the neuromuscular junction. Loss of Brp is an early hallmark of neuromuscular degeneration in Drosophila ${ }^{33}$.

In wild type, there is no change in Dlg over time (Fig. $4 \mathrm{a}, \mathrm{d}$ ), although minor changes in Dlg organization occur (Fig. 4a, inset).
By contrast, major changes are observed in the $S V I P^{K O}$. Dlg staining is slightly diminished compared to wild type at 1 week (Fig. 4b, d), but is nearly abolished by 4 weeks (Fig. 4b, d). In parallel, we observe that Brp puncta are dramatically reduced in number (Fig. 4b, bottom inset). We used Brp puncta as a proxy to calculate the length of the neuromuscular synapse and find a highly significant, age-dependent decrease in the SVIPKO (Fig. 4e). Importantly, both the change in Dlg and Brp are fully rescued by muscle-specific resupply of SVIP in SVIPKO (Fig. 4c-e). We conclude that loss of SVIP causes progressive degeneration of muscle membrane systems and neuromuscular synapses.

In order to extend our anatomical analyses, we used phalloidin as a marker of normal muscle actin organization. These analyses were performed in the adult abdominal muscles. As in the jump muscle (Fig. 4a-e), Dlg labels the t-tubule network, although it 

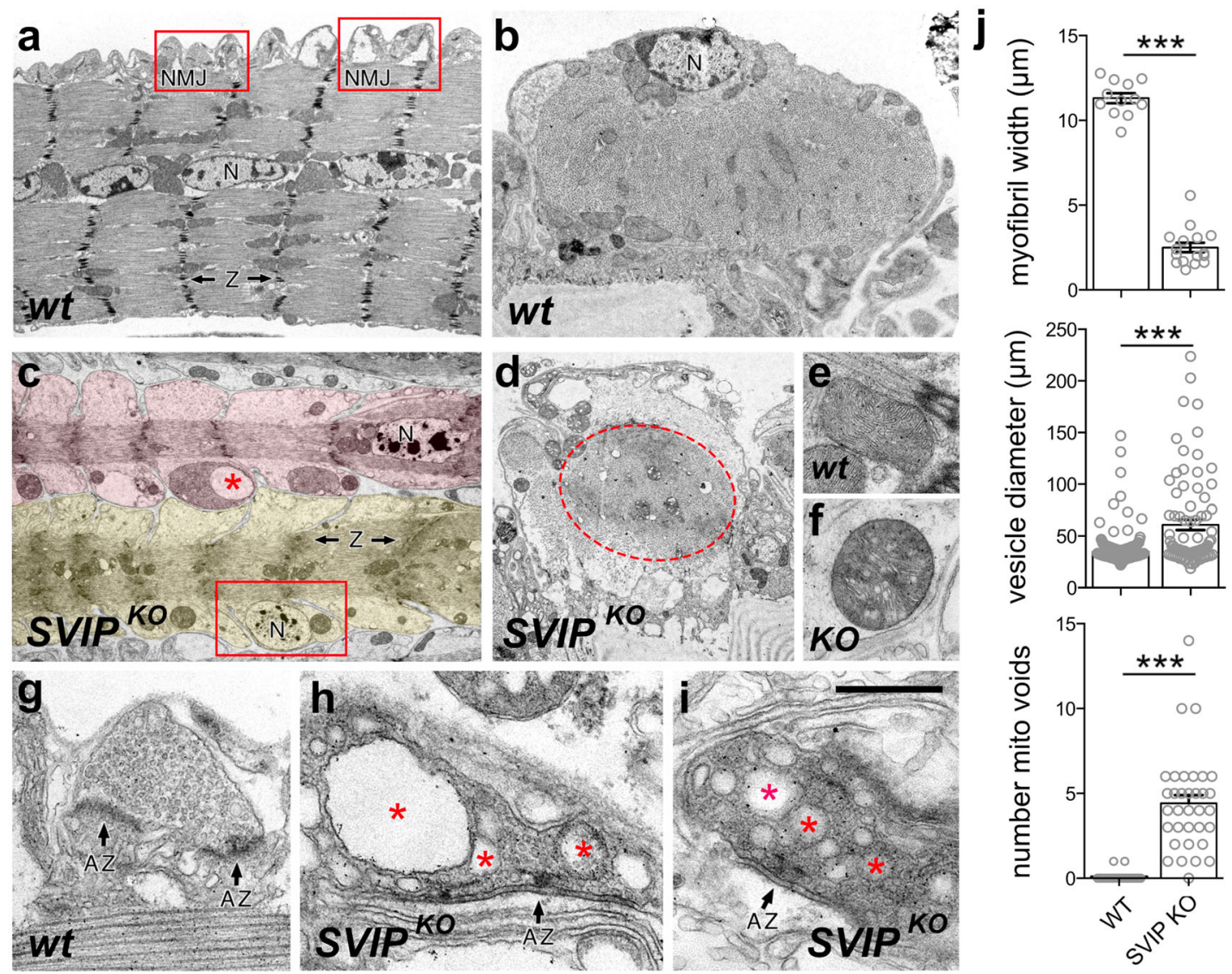

Fig. 5 Ultrastructural analyses of neuromuscular degeneration in SVIPKO. a Longitudinal section of wild-type (wt) adult abdominal muscle with NMJ indicated (red box), muscle nuclei ( $N$ ), and z-bands ( $Z$ ). The view encompasses a single muscle fiber. b Cross-section of wild-type (wt) adult abdominal muscle. $\mathbf{c}$ Longitudinal section of SVIPKO adult abdominal muscle. Two muscle cells exist in the field of view shaded pink and yellow. Altered mitochondria indicated (red asterisk). Peripheral nucleus $(N)$ red box is indicated. d Cross-section of SVIPKO adult abdominal muscle with a red dotted line indicating the outline of the muscle cell. e Representative mitochondria in wild type. f Representative mitochondria in SVIPKO (KO). $\mathbf{g}$ NMJ in wild type with active zones indicated (arrows). h, i NMJ in the SVIPKO. Arrows as in $\mathbf{g}$. Red asterisks highlight large vacuoles within the pre-synaptic nerve terminal. $\mathbf{j}$ Quantitation of myofiber width, vesicle diameter, and a number of mitochondrial voids. Scale bar represents a, c $5.0 \mu \mathrm{m}, \mathbf{b} 3.1 \mu \mathrm{m}, \mathbf{d} 4.9 \mu \mathrm{m}, \mathbf{e}, \mathbf{f} 1.1 \mu \mathrm{m}, \mathbf{g} 0.68 \mu \mathrm{m}, \mathbf{h} 0.45$ $\mu \mathrm{m}, \mathbf{i} 0.4 \mu \mathrm{m}$. Data presented as mean and SEM. Student's $t$-test for individual comparisons $\left({ }^{\star \star \star} p<0.001\right)$. See also Supplementary Fig. S5.

appears less striated in these muscles (Fig. 4f). In $S V I P K O$ animals, Dlg becomes completely diffuse, consistent with disruption of the t-tubule network (Fig. 4f, h). In addition, the phalloidin-labeled, actin-rich bands are broader and less well-defined than the $\operatorname{SVIPKO}$ (Fig. 4f, g). These data are consistent with the analyses of flight muscle and became the substrate for a more detailed analysis by electron microscopy (see next section).

Ultrastructural visualization of muscle wasting in SVIP knockouts. Muscle wasting, quantified by immunohistochemical techniques, correlates with impaired organismal motility, arguing for progressive neuromuscular degeneration. To assess neuromuscular degeneration in greater detail, we examined neuromuscular ultrastructure in adult Drosophila. In Fig. 5a, b, we present longitudinal (Fig. 5a) and cross-sections (Fig. 5b) of a single adult wild type, poly-nucleate muscle cell. Nuclei (N) are present in the central region of the muscle cell (Fig. 5a), occupying a peripheral location in cross-section (Fig. 5b). Prominent, well-organized Z-bands are apparent $(\mathrm{Z})$ with associated mitochondria. The neuromuscular synapses (Fig. 5a, red boxes, NMJ) are distributed along the muscle surface. Higher magnification of the NMJ $(G)$ reveals concentrations of synaptic vesicles at electron densities with characteristic T-bar structures that are diagnostic of sites of neurotransmitter release termed active zones (AZ in Fig. $5 \mathrm{~g}$ ).
The SVIPKO mutants show signs of severe wasting, impaired mitochondria morphology, and hallmarks of neuromuscular synapse degeneration. The diameter of individual muscle cells is dramatically reduced (Fig. $5 c, d, j$ ). Note that two muscle cells are shown (differentially colored in Fig. 5c) in the same physical space that is normally occupied by a single wild-type muscle cell (Fig. 5a). In SVIPKO mutants the acto-myosin cytoskeleton is greatly diminished with disorganized Z-bands (Z). The muscle nuclei $(\mathrm{N})$ are also variably positioned, consistent with impaired muscle cell health. Muscle mitochondria in SVIPKO mutants are altered with apparent vacuoles (Fig. 5c, red star). More commonly, however, mitochondria in the SVIPKO mutants show numerous, smaller voids within the intraorganellar membranes of unknown consequence (compare Fig. 5e, f; also see panel $j$ and Supplementary Fig. S5B, C). The mitochondria phenotypes we observe are recapitulated by muscle-specific over-expression of UAS-SVIPR24,25A (Supplementary Fig. S5D) emphasizing that this phenotype is likely related to the loss of SVIP-dependent VCP recruitment to the lysosomal membrane system. We also note that defects in mitochondria are rescued by muscle-specific over-expression of UAS-SVIP in SVIPKO (Supplementary Fig. S5A). Since the primary effect of SVIP muscle over-expression is the potent re-localization of VCP to lysosomal membranes, we propose that the observed mitochondria defects are secondary to the 
integrity of the lysosomal system, a possibility that is consistent with a role for VCP in mitophagy ${ }^{7}$.

Finally, neuromuscular synapses show ultrastructural hallmarks of degeneration, consistent with immunohistochemical observations (Fig. 4). Neuromuscular junctions (NMJ) are positioned at the muscle surface in cross-section in both wild type and in $S V I P^{K O}$ mutants (Fig. 5a). In $S V I P^{K O}$ mutants NMJ are characterized by a darkened appearance and the proliferation of large vesicles (asterisks $\mathrm{H}$, I; see also panel J for quantification) that are consistent with the appearance of enlarged endolysosomes and increased membrane internalization in other models of neuromuscular degeneration, both in Drosophila ${ }^{34}$ and mammalian systems ${ }^{35}$.

Muscle SVIP expression rescues neuronal degeneration in SVIPKO mutants. In mammalian systems, ALS and related neuromuscular degenerative disorders are characterized by synaptic degeneration, muscle wasting as well as degeneration of lower and upper motoneurons $s^{36}$. Thus, we tested whether degeneration is restricted to the NMJ, or whether it extends to motoneurons that participate in a simple motor circuit in the adult Drosophila central nervous system. We quantified neurodegeneration with single-cell resolution by using ShakB-Gal4 to drive expression of UAS-CD8-GFP in the tergotrochanteral motor neuron (TTMn) ${ }^{37}$ (Fig. 6a). The TTMn neuron is born and matures morphologically during pupal development (Supplementary Fig. S6A). Therefore, we quantified neuronal morphology during pupal development as well as during adult lifespan, comparing wild type to SVIPKO animals. Pupal development was staged as a percentage of total developmental time ${ }^{37}$. At $70 \%$ pupa development, the TTMn neurons are fully developed and there is no evidence of degeneration in SVIPKO animals (Supplementary Fig. S6B, C). However, in SVIPKO animals TTMn neurons rapidly begin to degenerate beginning at 1 day of adulthood and degeneration becomes progressively more severe over the first 30 days of adult life (Fig. $6 \mathrm{~b}-\mathrm{e}$ ). By 30 days, $100 \%$ of $S V I P^{K O}$ animals showed severe signs of motoneuron degeneration, including complete loss of dendrites and somas (Fig. 6b-e). In contrast, wild-type animals exhibited little to no signs of dendrite degeneration by 30 days of adulthood (Fig. 6b-e).

As an independent assessment of neurodegeneration, we assessed the function of a simple, well-defined neural circuit termed the giant fiber (GF) circuit (Fig. 6a) that impinges on the TTMn motoneuron ${ }^{38}$. Electrical stimulation of the GF neuron in the Drosophila head elicits activation of both the TTMn motoneuron (Fig. 6a, green) and the DLMn motoneuron (Fig. 6a, blue). It is possible to assess the integrity of this simple circuit by stimulating the GF and recording in the target musculature ${ }^{37}$ (Fig. 6a). Muscle responses are categorized as a failure if there is a complete absence of stimulus-time-locked muscle depolarization. Failures will occur if the neuromuscular synapse has completely uncoupled from the muscle, or if elements of the neural circuitry in the central nervous system have degenerated and broken the circuit. Complete synapse elimination at the NMJ is rarely observed (Fig. 4b, d, e), suggesting that complete failures likely reflect degeneration of elements within the central nervous system. Wild-type animals show a $100 \%$ success rate at 30 days in both target muscles (Fig. 6f, g). Young SVIPKO animals (2-5 days) have a $100 \%$ success rate, but by 30 days this has decreased to $0 \%$ success in the TTMn target muscle and to less than $40 \%$ success in the DLMn target muscle (Fig. 6f, g). These data are consistent with our anatomical analyses demonstrating severe motoneuron dendrite degeneration (Fig. 6b-e).

We next asked where SVIP is necessary to support the integrity of motoneurons in the CNS. Remarkably, we find that muscle- specific expression of UAS-SVIP completely rescues neurotransmission at the TTMn target muscle and doubles the success rate at the DLMn target muscle in SVIPKO animals (Fig. 6f, g). These results demonstrate that muscle-specific expression of UAS-SVIP is sufficient to rescue muscle health, NMJ integrity and it is also sufficient to improve motoneuron integrity. Thus, we suggest that SVIP-mediated recruitment of VCP to lysosomes in muscle can have profound cell non-autonomous consequences for the integrity of central neuronal circuitry and animal behavior. These data are consistent with a growing body of evidence that skeletal muscle proteostasis can have organism-wide effects on cellular health and organismal fitness ${ }^{39,40}$ (see "Discussion" section).

The ability of muscle SVIP expression to rescue motoneuron degeneration prompted us to determine the breadth of this effect. To do so, we quantified cell death in aged wild type and SVIPKO animals, as well as SVIPKO animals with muscle-specific SVIP rescue. Cell death was quantified by counting neuronal soma positively stained by ApopTag (see "Methods" section). In the region of the central nervous system where the majority of motoneurons reside that innervate the limbs, abdominal muscles, and flight muscles (termed ventral nerve cord; shown in Fig. 6) aged SVIPKO animals show a significant increase in cell death that is fully rescued by muscle-specific expression of SVIP (Fig. 6h, i). By contrast, although significantly elevated cell death was observed within the neural circuitry of the head and the visual system in the aged $S V I P K O$, this cell death was not rescued by muscle-specific expression of SVIP (Fig. 6j, k). These data agree with the muscle-specific, cell non-autonomous rescue of motoneurons at the single-cell level (Fig. 6b-g). These data also suggest that the restoration of muscle health by muscle-specific SVIP expression in $S V I P^{K O}$ is achieved by localized trophic support of motor circuitry rather than an organism-wide effect.

Identification of a $V C P$ disease-associated mutation that abolishes VCP-SVIP binding. To this point, we have focused on the necessity of the SVIP gene. We next sought to connect SVIP activity to human disease-relevant mutations in the VCP gene. We hypothesize that pathogenic mutations in $V C P$ could, in some instances, directly impair the VCP-SVIP interaction, precluding SVIP-dependent VCP recruitment to lysosomes, causing multisystem failure. To test this hypothesis, we biochemically screened 10 conserved, disease-relevant, missense mutations in VCP (Supplementary Table 1) for loss of SVIP binding. Four mutations reside in the $\mathrm{N}$-terminal protein-protein interaction domain, four reside in the D1 ATPase domain and two reside in the first linker region (Fig. 7a). Mutations in the N-terminal region had the largest effect of reducing SVIP-VCP affinity (Fig. 7b-e). The most dramatic effect was observed in the P134L mutant, which reduced SVIP-VCP affinity more than 30 -fold (Fig. 7b, c).

Given the marked reduction in SVIP-VCP binding caused by the $V C P^{P 134 L}$ mutation, we sought to determine the in vivo consequences of this mutation, comparing them to the effects of the SVIPKO. To better mimic disease, we used CRISPR to knockin the $V C P^{P 134 L}$ mutation at the endogenous $V C P$ locus. An sfGFP tag was fused to the mutant transgene to enable

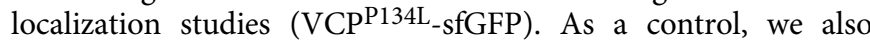
generated a knock-in of wild-type VCP fused to sfGFP (VCPWT sfGFP). While we were able to obtain homozygous $V C P^{W T} T_{-s f G F P}$ adult flies, we failed to obtain any homozygous $V C P^{P 134 L_{-s f G F P}}$ adults, suggesting that the mutation is homozygous lethal. Given that IBMPFD is autosomal dominant, $V C P^{P 134 L}$ heterozygotes should genetically mimic the human disease and we further characterized the defects associated with the heterozygous mutant. 
a

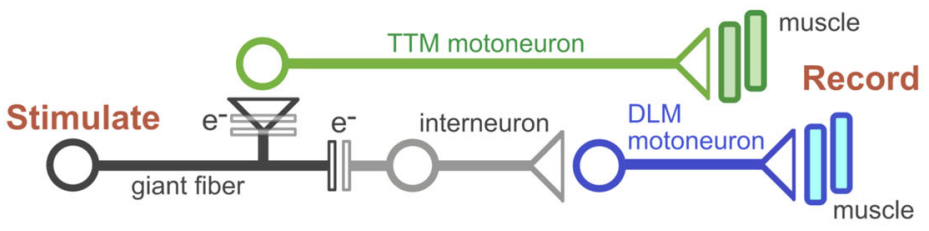

b
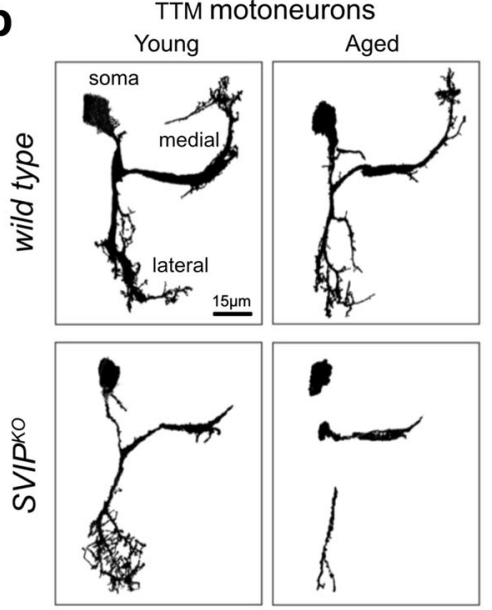

f wild type SVIPKo rescue aged young aged aged
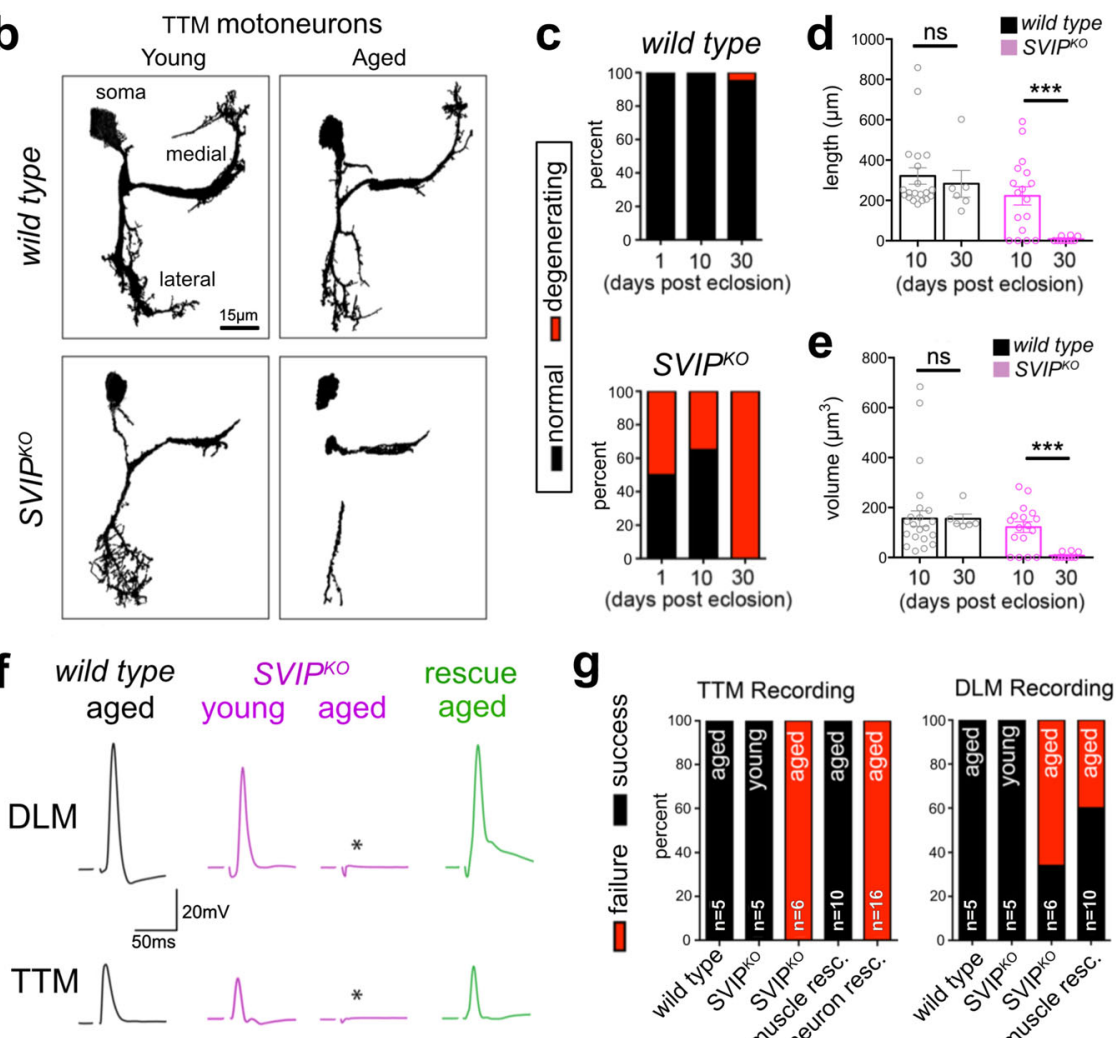

g

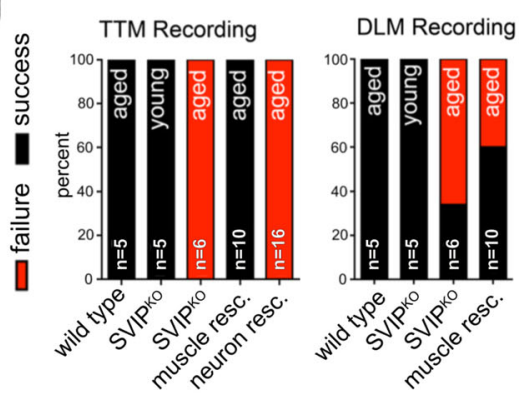

h

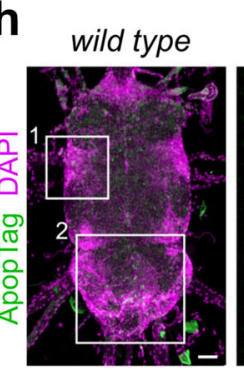

i

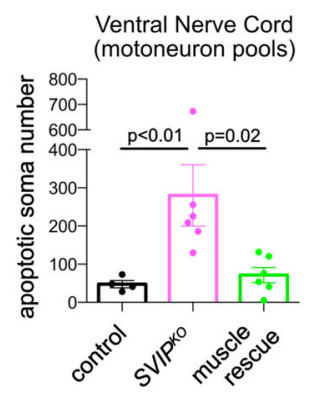

muscle
rescue

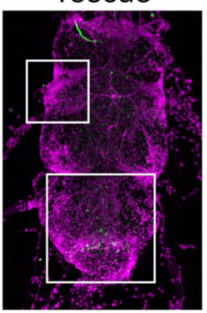

head

j

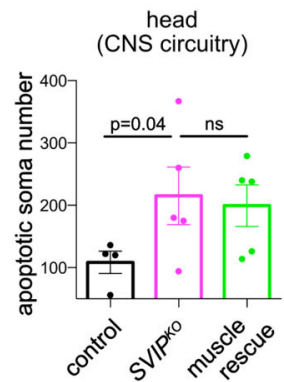

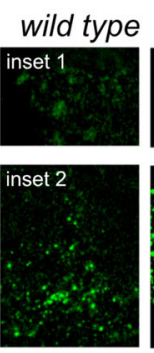

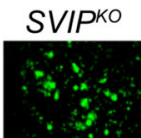

rescue
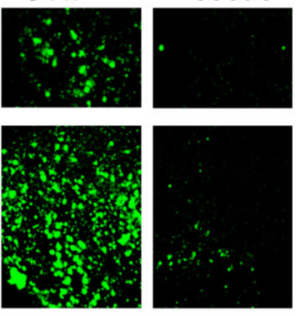

K $\quad \begin{gathered}\text { Optic Lobes } \\ \text { (sensory circuitry) }\end{gathered}$

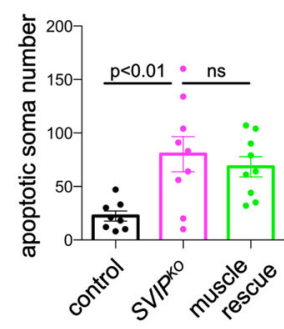

VCP protein self-oligomerizes into a hexamer. Since we are analyzing $V C P^{P 134 L}$ heterozygous mutants, we wanted to examine whether the P134L mutant protein could assemble with wild-type VCP normally in vivo. To assess this, we performed coimmunoprecipitations. The epitope-tagged $\mathrm{VCP}^{\mathrm{P} 134 \mathrm{~L}_{-} \text {sfGFP }}$ allows easy resolution of the mutant protein compared to wild- type VCP by gel electrophoresis. VCPWT-sfGFP heterozygous flies were used to control for the sfGFP tag interfering with VCP

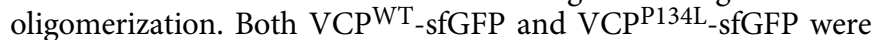
able to co-immunoprecipitate wild-type VCP (Fig. 7f), indicating that the P134L mutation does not prevent VCP selfoligomerization. Since VCP hexamers should consist of both 
Fig. 6 Neurodegeneration in the SVIPKO and cell non-autonomous muscle rescue. a Diagram of the giant fiber neural circuit inclusive of the giant fiber residing in the animal head (gf) the PSI interneuron (int, gray), the DLM (blue), and TTM (green) motoneurons and muscles. Synaptic connections are indicated as electrical (e-, parallel lines), chemical (triangle), or mixed (superimposition of triangle and parallel lines). b Representative images of wild type and SVIPKO TTM motoneurons at 10 days (young) and 30 days (aged). c Quantification of the percentage of total visualized motoneurons that showed evidence of degeneration (see main text) (WT 10 days $n=20$ cells examined over 10 independent animals, SVIPKO 10 days $n=17$ cells examined over nine independent animals, WT 30 days $n=6$ cells examined over 5 independent animals, SVIPKO 30 days $n=9$ cells examined over 9 independent animals). d, e Quantification of TTM dendrite morphology including primary dendrite length (d), dendrite volume (e). f Representative recordings from muscles innervated by the DLM motoneuron (top) or TTM motoneuron (bottom). Ages as in $\mathbf{b}$. Stimulus artifact removed for clarity. $\mathbf{g}$ Quantification of NMJ recordings for indicated muscles and genotypes (TTMn: WT old $n=5$ animals, SVIPKO young $n=5$ animals, SVIPKO old $n=6$ animals, muscle rescue old $n=10$ animals, MN rescue $n=16$ animals; DLM: WT old $n=5$ animals, SVIPKO young $n=5$ animals, SVIPKO old $n=6$ animals, muscle rescue old $n=10$ animals). h Apoptag (green) and DAPI (magenta) staining of ventral nerve cords in the genotypes indicated. Insets are shown at right. Scale bar $70 \mu \mathrm{m}$. i-k Quantification of apoptotic soma in the ventral nerve cord (i control $n=4$ animals, SVIPKO $n=4$ animals, rescue $n=6$ animals), head ( $\mathbf{j}$ control $n=4$ animals, SVIPKO $n=4$ animals, rescue $n=6$ animals) (j) and optic lobe (k, subset of the head; control $n=8$ optic lobes examined over four independent animals, SVIPKO $n=9$ optic lobes examined over five independent animals, rescue $n=9$ optic lobes examined over 5 independent animals). See also Supplementary Fig. S6. Data presented as mean and SEM. Student's $t$-test for individual comparisons $\left({ }^{\star} p<0.05 ;{ }^{\star \star} p<0.01 ;{ }^{\star \star \star} p<0.001\right)$. ANOVA for multiple comparisons $\left({ }^{\star} p<0.05 ;{ }^{\star \star} p<0.01 ;{ }^{\star \star *} p<0.001\right)$.

wild-type and mutant VCP protein, we tested whether VCP mutant heterocomplexes displayed reduced binding to SVIP in vivo. Indeed, the VCP heterocomplex immunoprecipitated significantly less SVIP compared to a wild-type VCP complex (Fig. $7 \mathrm{~g} ; p<0.05$ ). Given this result, we examined lysosomes in the $V C P^{P 134 L}$ mutant animals by imaging Spin-RFP. Similar to what we observed in SVIPKO animals, the dynamic lysosomal network is significantly disrupted (Fig. 7h). We document significant defects in tubule length, tubule density, and tubule complexity (junctions per tubule) (Fig. 7h). Moreover, we note significantly less localization of $\mathrm{VCP}^{\mathrm{P} 134 \mathrm{~L}_{-}}$-sfGFP at lysosomes (Supplementary Fig. S7A). Thus, a disease-causing mutation in VCP (P134L) impairs both SVIP binding and the lysosomal network in muscle.

\section{VCPP134L mutant protein aggregates in an age-dependent} manner and causes degeneration. We find that endogenously expressed VCP localizes to tubular structures around the nucleus and in the cytoplasm (Fig. 8a). In young adult muscles (day 1),

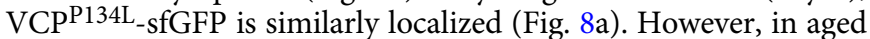

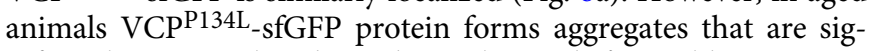
nificantly more abundant than observed for wild-type VCP (Fig. 8a, b). To further explore whether the $\mathrm{VCP}^{\mathrm{P} 134 \mathrm{~L}}$ mutant protein has an increased propensity to aggregate, we performed an in vivo aggregation assay. We found that application of a VCP

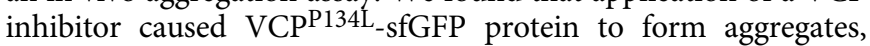

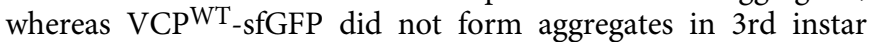
larval muscles (Supplementary Fig. S7b). It appears that age and cellular stress can trigger the formation of aggregates that contain the $\mathrm{VCP}^{\mathrm{P} 134 \mathrm{~L}}$ mutant protein. It is possible that the $\mathrm{VCP} \mathrm{P}^{\mathrm{P} 134 \mathrm{~L}}$ mutant protein is aggregation prone or $\mathrm{VCP}^{\mathrm{P} 134 \mathrm{~L}}$ recognizes aggregates that form due to impaired $\mathrm{VCP}^{\mathrm{P} 134 \mathrm{~L}}$ ability to target the lysosome. In either case, our data are consistent with observations in human IBMPFD patients where protein aggregates stained positively for VCP protein ${ }^{10}$.

Finally, we examined the organismal defects associated with the $V C P^{P 134 L}$ mutation (Fig. 8c-f). The lifespan of $V C P^{P 134 L}$ mutants was modestly, but significantly, reduced compared to VCPWT (Fig. 8e, $p<0.001$ ) and displayed progressive mobility defects (Fig. 8f). We then examined neuronal function at the DLM neuromuscular junction in adult Drosophila. It is well established that this synapse can reliably evoke muscle depolarization when stimulated at $100 \mathrm{~Hz}$. When we repeated this stimulation paradigm in aged control animals, the motor neuron could evoke a response for every stimulus (Fig. 8c). However, $V C P^{P 134 L}$ aged mutant animals exhibited a $70 \%$ failure rate (Fig. $8 \mathrm{c}$ ). Direct stimulation of the motoneuron evokes successful muscle depolarization, demonstrating that failures do not originate at the NMJ (Fig. 8c, d). Rather, this phenotype is consistent with degeneration of central processes, such as the motoneuron dendrite degeneration in the $S V I P^{K O}$ as well as widespread cell death in the $S V I P^{K O}$ (Fig. 6). Finally, we quantified NMJ integrity based on the release site quantification (anti-Brp staining). We find a significant reduction in Brp puncta in the $V C P^{P 134 L}$ aged mutant compared to control and numerous boutons that completely lack Brp puncta (Fig. 8g, h), indicative of synapse degeneration ${ }^{33}$. Note, however, that there was limited evidence of complete synapse elimination, consistent with ongoing synapse degeneration and continued neurotransmission from nerve to the muscle. Taken together, the organismal and cellular defects observed in $V C P^{P 134 L}$ mirror many of the phenotypes in the SVIPKO.

Identification of a human SVIP mutation. In order to explore additional connections between SVIP and human disease, we screened a cohort of sporadic FTD patients $(n=341)$, with whole-genome sequencing data from the UCSF Memory and Aging Center (MAC), for putative pathogenic variants in SVIP. We found one potentially deleterious heterozygous nonsynonymous single nucleotide variant (SNV) in SVIP at genomic coordinates Chr11:22,844,669. This SNV results in a C to T substitution at nucleotide 230 in the coding sequence (NM_148893: c.230 C>T) and was subsequently confirmed with Sanger sequencing. This variant causes a serine to leucine substitution, p.S77L, with the substituted amino acid being highly conserved from Drosophila to human (refer back to Fig. 1b). Importantly, we did not find the c. $230 \mathrm{C}>\mathrm{T}$ or any other nonsynonymous SVIP variants in 106 clinically normal, healthy older controls or 318 early-onset Alzheimer's disease patients from the UCSF MAC cohorts. Further, to the best of our knowledge, this variant has not been reported in public whole-genome and/or exome sequencing databases, including the Genome Aggregation Database (gnomAD) and Exome Aggregation Consortium (ExAC), suggesting that this variant is extremely rare in the general population. Finally, this patient was negative for known pathogenic mutations in the following genes: APP, C9ORF72, CHMP2B, FUS, GRN, MAPT, PSEN1, PSEN2, and TARDBP.

SVIPS82L disrupts lysosome structure and function in Drosophila. To examine potential defects associated with the human S77L mutation, we first examined SVIP-VCP binding. We generated a GST fusion protein of Drosophila SVIP harboring the orthologous mutation (S82L, see Fig. 1b for reference) and found 
a

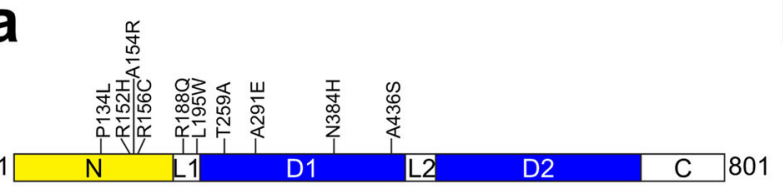

b

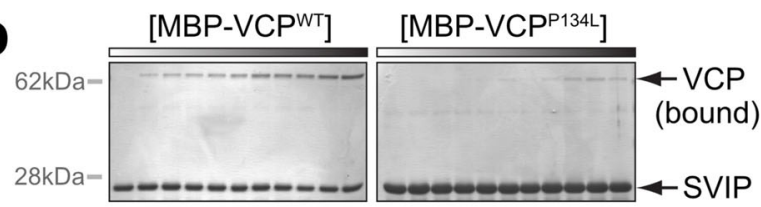

C

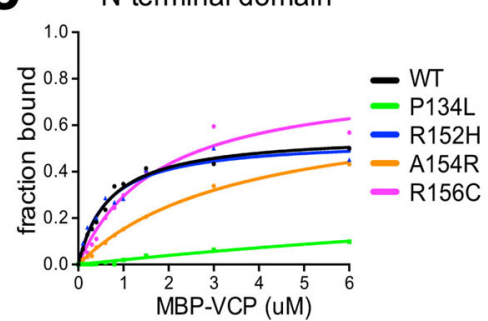

mutation affinity to SVIP

\begin{tabular}{|c|c|}
\hline WT & 0.70 \\
\hline P134L & 21.64 \\
\hline R152H & 0.64 \\
\hline A154R & 3.61 \\
\hline R156C & 1.77 \\
\hline
\end{tabular}

f

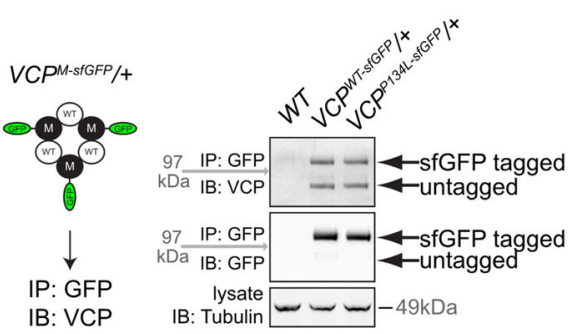

IB: VCP

IB: Tubulin $\sim-\sim-49 \mathrm{kDa}$ d

L1 linker

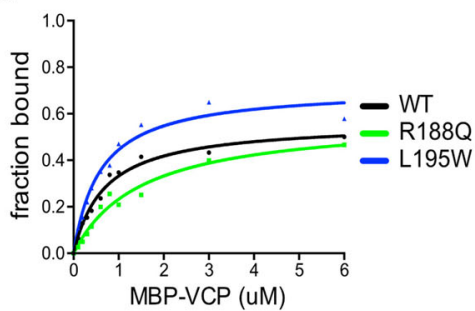

mutation affinity to SVIP

\begin{tabular}{|c|c|}
\hline WT & 0.70 \\
\hline R188Q & 1.50 \\
\hline L195W & 0.60 \\
\hline
\end{tabular}

e

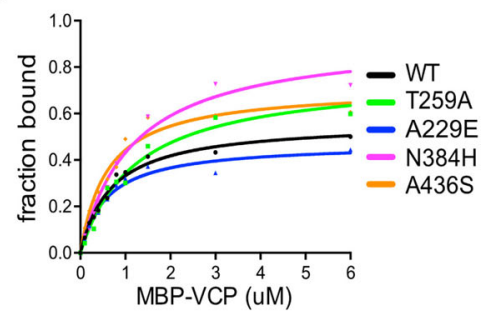

mutation affinity to SVIP
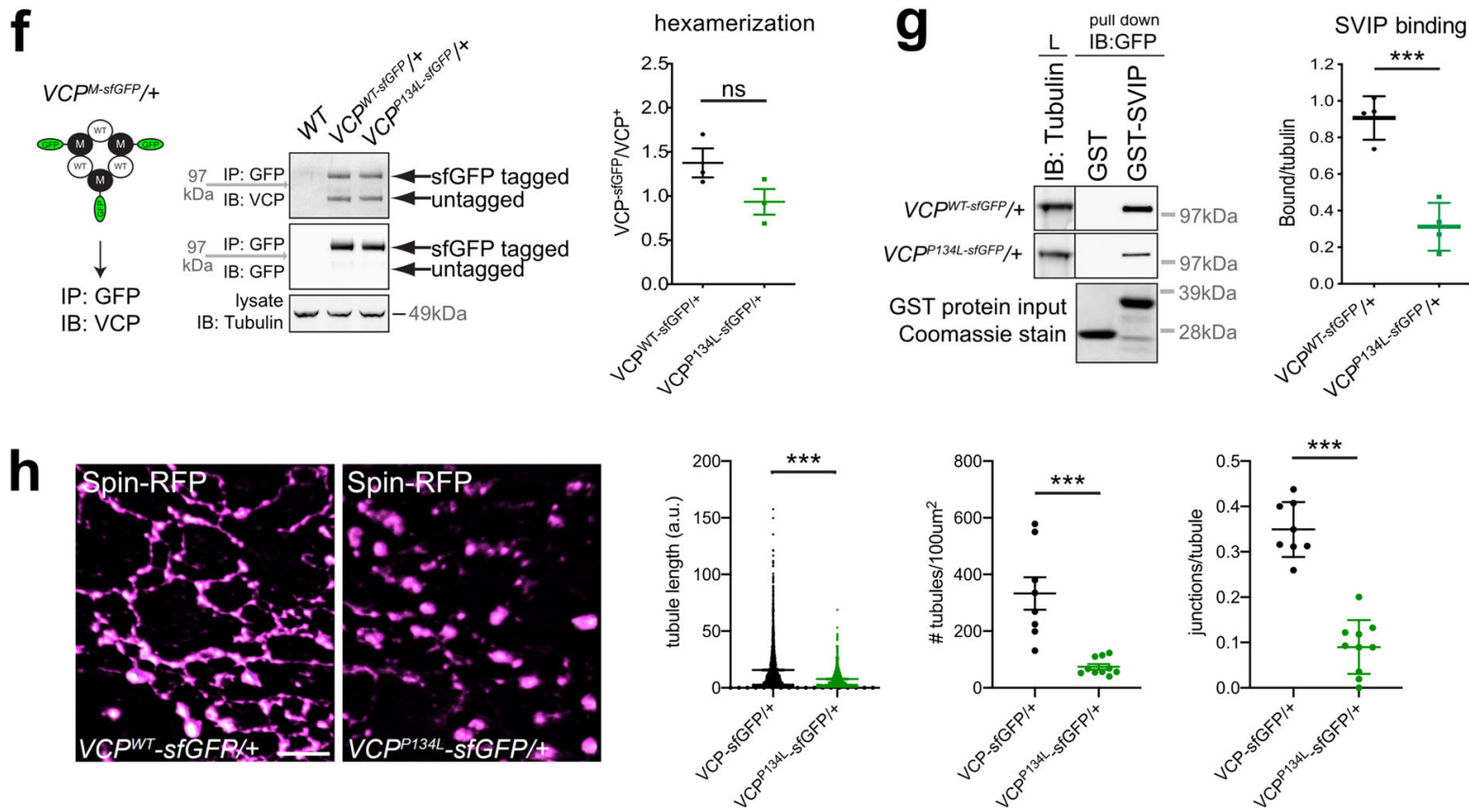

Fig. 7 VCP disease mutation abrogates SVIP-VCP interaction. a Schematic of VCP with relative positions of disease-relevant mutations. $\mathbf{b}$ In vitro binding titration assay of GST-SVIP with MBP-VCPWT and MBP-VCPP134L recombinant proteins. c-e Binding curves of GST-SVIP with each MBP-VCP mutant as indicated. Affinities are listed in the tables for each mutant. $\mathbf{f}$ Co-immunoprecipitation of endogenously expressed VCP and VCP-sfGFP (transheterozygote). Diagram of hexameric VCP protein with proposed SVIP (green) binding at left. Quantification at right. ( $n=3$ independent experiments). $\mathbf{g}$ In vitro binding assay with recombinant GST-SVIP and VCP-sfGFP protein lysates extracted from whole flies. Quantitation of the amount of VCP-sfGFP protein bound to GST-SVIP. ( $n=3$ independent experiments). h Representative images of Spin-RFP in VCPwt and VCPP134L mutants. Quantification at right. ( $n=8$ muscle cells examined over four independent animals). Data presented as mean and SEM. Student's $t$-test for individual comparisons $\left({ }^{\star} p<0.05 ;{ }^{\star \star} p<0.01 ;{ }^{\star \star \star} p<0.001\right)$.

that SVIPS82L can still bind VCP in vitro (Supplementary Fig. S8). Next, we generated a Drosophila SVIPS82L transgene. When SVIPS82L is over-expressed in an SVIP heterozygous knockout $(S V I P K O /+)$ background we find that lysosomal tubules are no longer visible and lysosomal dynamics are severely perturbed (Fig. 9a; Supplementary Movies 1 and 2). Finally, an SVIP $882 L$ knock-in, when tested in a lifespan assay, also showed resistance to the effects of tunicamycin feeding, similar to SVIPKO mutants
(Fig. 9b). Thus, although SVIPS82L can still bind VCP in vitro, the mutant protein is sufficient to dominantly disrupt tubular lysosomes and affect animal physiology.

Finally, we imaged the mCh-GFP-Atg8a autophagy sensor to compare the proficiency of autophagosome-lysosome fusion in animals over-expressing SVIPWT or SVIPS82L in the SVIP heterozygous knockout background $(S V I P K O /+)$ (Fig. 9c, d). When SVIPWT is over-expressed, we observed little to no GFP 
a
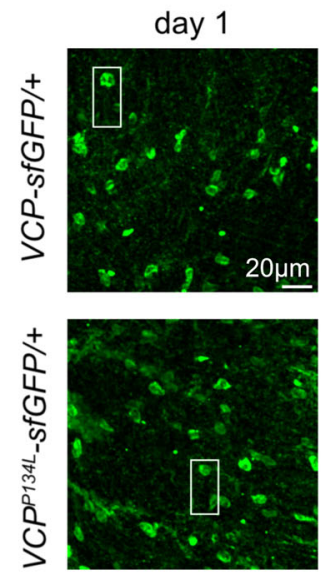

C

VCP-sfGFP/+ day 30

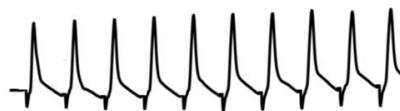

$0 \%$ failure rate

VCP ${ }^{P 134 L}$-sfGFP/+ day 30

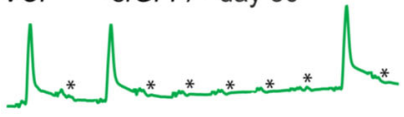

$70 \%$ failure rate
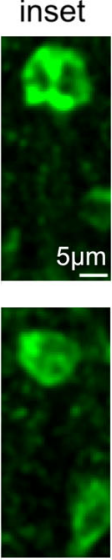
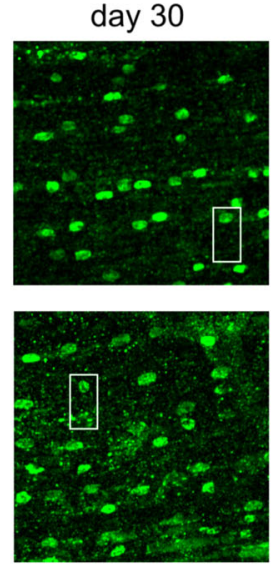
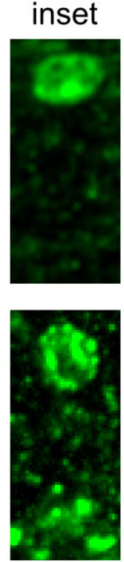

b

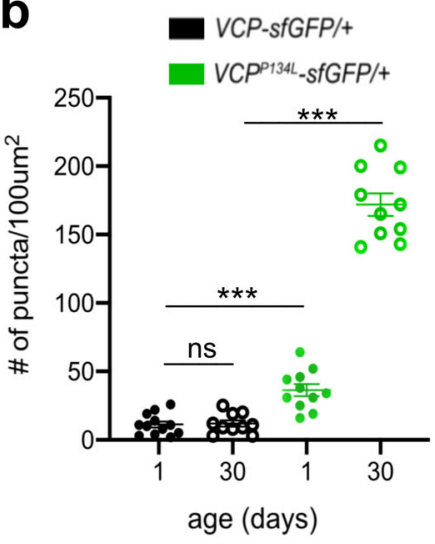

f

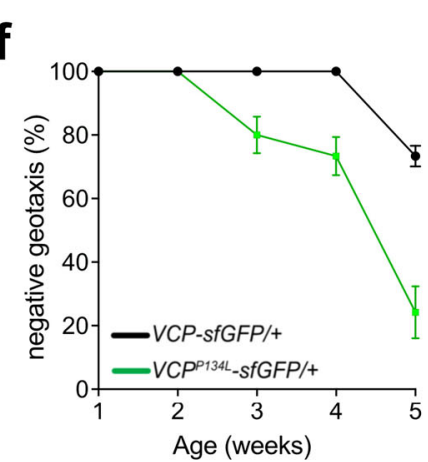

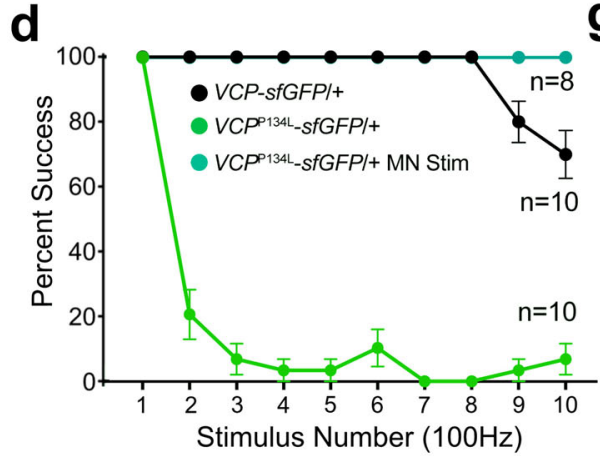
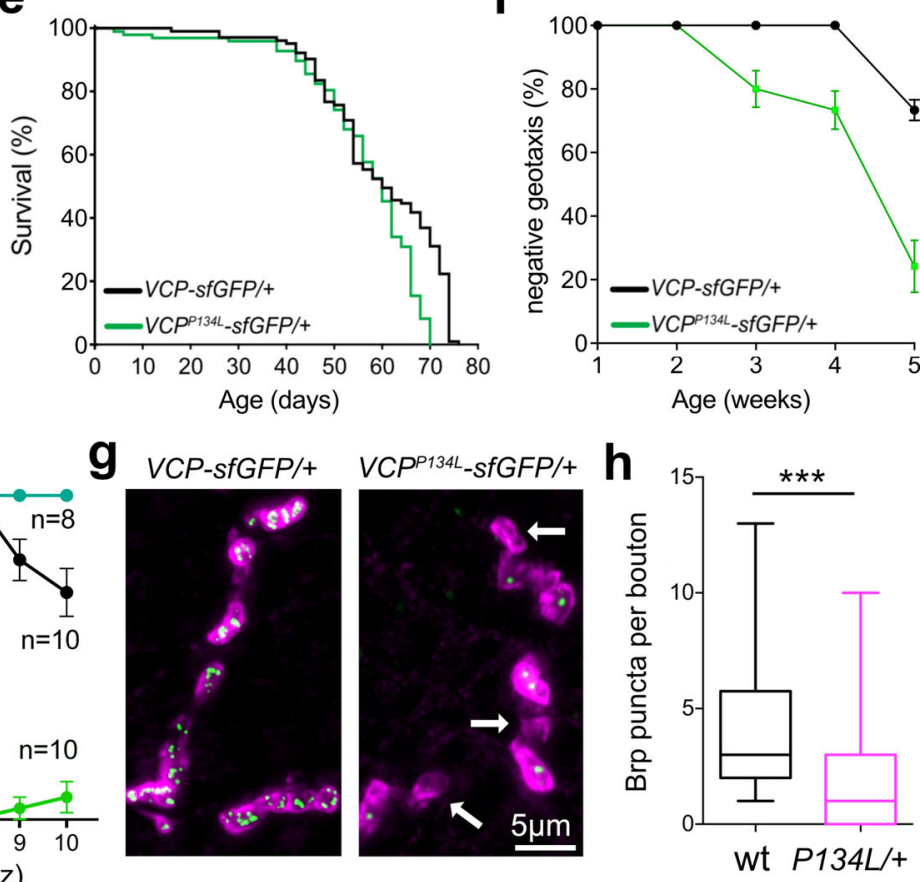

Fig. 8 VCPP134L knock-in mutants cause VCP aggregation and functional degeneration. a Localization of endogenously expressed VCPWT-sfGFP and VCPP134L_sfGFP in young and aged TTM muscles. b Quantification of puncta density for data in a. Data presented as mean and SEM. c Representative traces recorded from the DLM muscle in wild type and VCP ${ }^{P 134 L}$. Failures are indicated by asterisks. Average failure rates are indicated below each trace ( $n=10$ animals for each genotype), stimulus artifact removed for clarity. $\mathbf{d}$ Quantification of data in $\mathbf{c}$ for the genotypes indicated. Stimulation was achieved either through activation of the giant fiber (a) or direct motoneuron stimulation (MN stim). e Lifespan of VCPWT-sfGFP $(n=103)$ and VCPP134L-sfGFP ( $n=$ 97). $\mathbf{f}$ Negative geotaxis assay for genotypes at indicated ages. Data from three independent experiments with 20 animals each are presented as mean and SEM for each age point. $\mathbf{g}$ Representative images of NMJs stained with Brp (green) and DLG (magenta). h Quantification of Brp per bouton. Data presented in a box and whisker plot (minimum, 1st quartile, median, 3rd quartile, and maximum). Scale bars indicated on images. See also Supplementary Fig. S7. ANOVA for multiple comparisons $\left({ }^{\star} p<0.05 ;{ }^{\star \star} p<0.01 ;{ }^{\star \star *} p<0.001\right)$.

fluorescence where mCherry fluorescence was observed, indicating that autophagosomes are efficiently fusing with lysosomes. However, when $m C h-G F P-A t g 8 a$ is co-expressed with SVIPS82L, we observed a significant increase in GFP fluorescence, indicating autophagosome-lysosome fusion impairment (Fig. 9c, d). Collectively, these data suggest that the $\operatorname{SVIP}(S 82 L / S 77 L)$ mutation is pathogenic in Drosophila muscle.

\section{Discussion}

IBMPFD is characterized by degeneration of tissues, including the neuromuscular junction and central neurons, causing similar symptoms associated with $\mathrm{ALS}^{3,4}$, Parkinson's ${ }^{7,8}$, and $\mathrm{FTD}^{9}$. Although IBMPFD is a comparatively rare disease, it has the potential to inform about generalized mechanisms that drive neurodegeneration more broadly. Here, we define SVIP as a cytoplasmic co-factor that is responsible for the translocation of VCP to a dynamic, tubular-lysosomal system in muscle. The importance of SVIP is underscored by our phenotypic analysis of SVIP knockouts and VCP transgenic knock-in mutants that disrupt the SVIP-VCP interaction. The SVIPKO shows a progressive, degenerative phenotype in muscle and motoneurons that is similar to that observed in disease-relevant VCP mutants as well as patients with IBMPFD and ALS. Degeneration is associated 
a

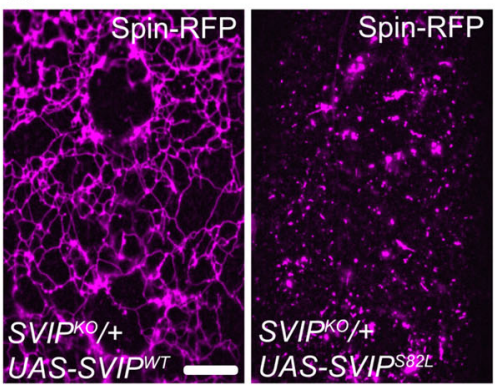

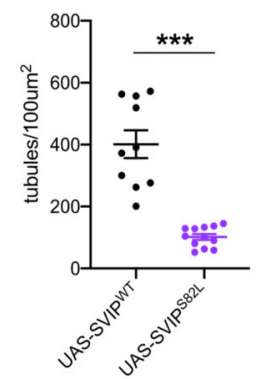

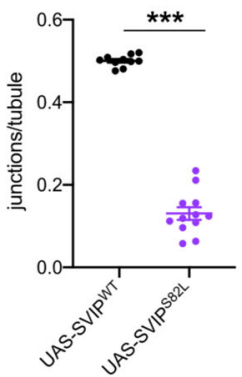

b

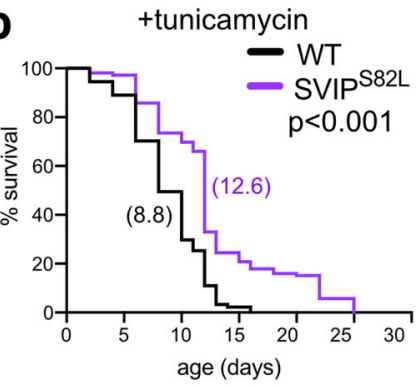

C

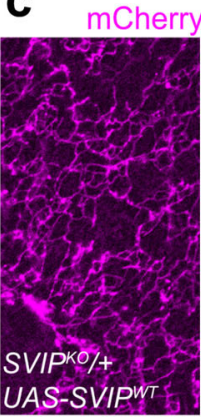

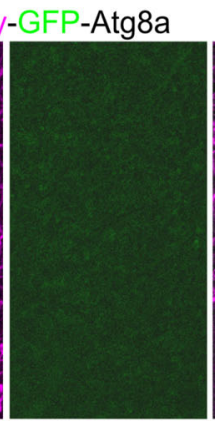
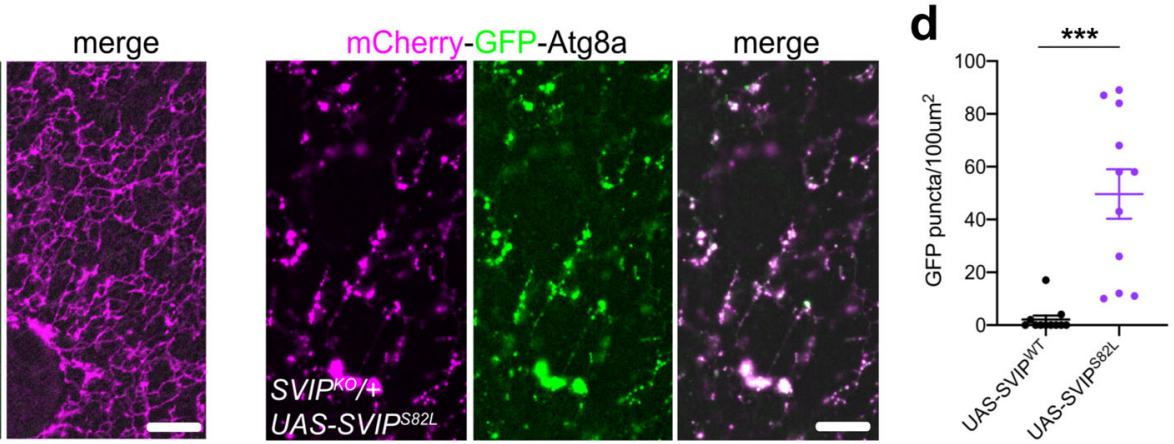

Fig. 9 SVIP variant that disrupts lysosome integrity and function. a Representative images of Spin-RFP (lysosomes) in 3rd instar larval Drosophila muscles for the genotypes indicated. At right, quantification of tubule density (tubules $\left./ 100 \mu \mathrm{m}^{2}\right)$ and junctions per tubule. $(n=10$ muscle cells examined over five independent animals). b Lifespan of WT $(n=91)$ and SVIPS82L $(n=106)$ flies fed tunicamycin $(p<0.0001)$. c, e Representative images of mCherry-GFPAtg8a (autophagy sensor) in 3rd instar larval Drosophila muscles for the genotypes indicated. d Quantification of data from c. ( $n=10$ muscle cells examined over five independent animals). Scale bars, $5 \mu \mathrm{m}$. See also Supplementary Fig. S8 and Supplementary Movies S1 and S2. Data presented as mean and SEM. Student's $t$-test for individual comparisons $\left({ }^{\star \star \star} p<0.001\right)$.

with the disintegration of intracellular membrane systems and disrupted mitochondria. We show that these phenotypes extend to progressive deficits in organismal behavior and diminished lifespan.

Regulated deployment of VCP by SVIP and related co-factors. Our data speak to the possibility that VCP is deployed to different organelles based upon stress-related or cell-specific needs (Fig. 1a). This is consistent with the prior demonstration that VCP is recruited to mitochondria following pharmacological poisoning of oxidative phosphorylation with cyanide $m$-chlorophenyl-hydrazone (CCCP ${ }^{7}$. Given the diverse activities of VCP, it was initially surprising that targeting the majority of VCP to lysosomes in muscle could have a beneficial outcome for organismal lifespan. Different cell types may routinely confront different stressors and emphasize different VCP co-factors. The fact that muscle lysosomes exist as an expansive, dynamic tubular network implies the importance of lysosomes to muscle biology and could underscore why SVIP knockouts have a particularly profound effect on muscle, with ensuing ALS-like degeneration of the neuromuscular junction and motoneuron dendrites.

SVIP controls organismal health and lifespan under conditions of lysosomal stress. Our data suggest that SVIP is a contextspecific survival factor. We demonstrate that SVIP over-expression, which is sufficient to drive VCP to lysosomes, is also sufficient to enlarge the dynamic tubular-lysosomal network in muscle. Musclespecific SVIP over-expression doubles lifespan when organisms are reared on food containing a lysosomal stressor, BafA. In one scenario, the loss of lysosomal $\mathrm{pH}$ caused by BafA is counteracted by the expansion of the tubular-lysosomal system, caused by SVIP over-expression. However, it is possible that the size and extent of the lysosomal system are less relevant compared to the increased abundance of VCP at the lysosomal compartment. In either case, SVIP-dependent recruitment of VCP to muscle lysosomes dramatically extends lifespan under conditions of lysosomal stress. Future efforts might be directed toward assessing whether SVIP overexpression can ameliorate the pathology and etiology of diseases associated with impaired lysosomal integrity. For example, lysosomal storage diseases are a group of $\sim 50$ rare metabolic disorders that primarily affect children, causing lethality within months or a few years of age.

It is important to emphasize that, while blocking SVIPmediated VCP localization to lysosomes (SVIP knockout) is maladaptive if animals are fed bafilomycin-A, it is beneficial if animals are fed tunicamycin, stressing the ERAD pathway. We interpret this effect as being a consequence of more VCP being available to the ER system in the absence of lysosomal localization, consistent with the initial identification of SVIP as a negative regulator of ERAD ${ }^{24}$. Thus, SVIP can be considered a context-specific survival factor. This finding is also consistent with our model regarding the dynamic deployment of a limited VCP resource to sites of cellular stress (Fig. 1a).

It is also worth noting that reduced proteostasis is a general feature of muscle aging and degeneration ${ }^{41}$. Indeed, proteostasis mechanisms naturally decline with age ${ }^{42}$ and are thought to be a major contributing factor to the onset of neurodegenerative diseases ${ }^{43-45}$. The predominant cellular degradation sites for clearing and recycling cellular waste products are lysosomes. Enhancing autophagy-lysosome-mediated proteostasis either pharmacologically or by caloric restriction has been shown to increase organismal healthspan in multiple species, including worms, flies, and mammals ${ }^{46}$. 
The sites of action for the beneficial effects of caloric restriction and enhanced autophagy-lysosome mediated proteostasis are under investigation. Intriguingly, there have been multiple examples of muscle proteostasis influencing the broader health of an organism. In particular, disrupting proteostasis in C. elegans muscles causes upregulation of the heat shock response system in distant tissues via the expression of specific chaperone proteins ${ }^{39}$. In Drosophila, the muscle-specific expression of FOXO enhances organismal viability ${ }^{40}$. These "tele-proteostasis" mechanisms may promote organismal robustness in a stressful environment by communicating the existence of proteotoxic stress to surrounding, healthy tissue, enabling a preparatory response. In essence, this is a type of feed-forward stress signaling mechanism that promotes organismal robustness.

Models of IBMPFD. Current Drosophila models of IBMPFD are based upon transgene over-expression, with unknown consequences on the normal regulation of VCP protein throughout the cell. In particular, it has been proposed, based on transgenic over-expression models, that IBMPFD is associated with adverse VCP gain of function activity ${ }^{22}$. By extension, it was proposed that VCP inhibitors might be beneficial to disease progression and human health ${ }^{22}$. However, the complexity of VCP signaling, acting at many sites within the cell (Fig. 1a) seems to argue for other therapeutic approaches.

To facilitate future therapeutic approaches, we have generated several mutants and transgenic animals that can be considered Drosophila models for exploration of IBMPFD. The VCPP134L knock-in and the SVIPKO specifically target the SVIP-dependent recruitment of VCP to lysosomes. It is interesting that several pathogenic VCP mutations do not affect SVIP binding. Yet, there is clear phenotypic overlap comparing the loss of $\mathrm{VCP}^{16}$ to the $V C P^{P 134 L}$ knock-in and the SVIPKO. Future work will be required to determine how the diversity of pathogenic $V C P$ mutations are linked to autophagosomal-lysosomal dysfunction. Regardless, our data suggest that targeting the interface of VCP with specific cofactors such as SVIP may allow modulation of VCP activity at specific intracellular sites, with the potential to ameliorate mutation-dependent diseases without compromising VCP activity globally throughout the cell.

Finally, we identify a human patient with FTD harboring a mutation in SVIP and demonstrate that this mutation causes pathological defects in Drosophila muscle lysosomes and impairs autophagy that parallels the phenotypes of our other Drosophila disease models. The fact that SVIP is a small gene makes it unlikely that a substantial patient population will harbor SVIP mutations, possibly precluding the determination of whether SVIP mutations are actually causal for human disease. None-theless, the fact that the patient-derived mutation is pathogenic in Drosophila muscle, phenocopying other disease models, argues that SVIP may be relevant to the human condition and encourages further investigation into the potential benefits of SVIP over-expression as a potential suppressor of human disease.

\section{Methods}

Fly stocks and molecular cloning methods. The following transgenic fly stocks were generated in this study: SVIP-sfGFP (endogenous gene replacement), $d V C P$ sfGFP (endogenous gene replacement), SVIPKO, SVIPS82L, UAS-SVIP, UAS-SVIPmCherry, UAS-SVIPR24,25A-mCherry, UAS-FKBP F37V $-S p i n-m C h e r r y, U A S-F R B-$ dVCP-Venus, UAS-SVIPS82L. Drosophila VCP, SVIP, and Spin cDNAs were obtained by amplifying from DGRC (Drosophila Genomics Resource Center, Bloomington, IN) clones GM02885, GH02734, and AT25382, respectively. The $F K B P^{F 37 V}$ and $F R B$ constructs were purchased from Addgene. The cDNAs were cloned into the Gateway pENTR vector (ThermoFisher) and subsequently cloned into destination vector pTWV obtained from the Drosophila Gateway Vector Collection (Carnegie Institution, Baltimore, MD). Injection services of BestGene were used to generate transgenic flies. UAS-GFP-mCherry-Atg8a stock was purchased from the Bloomington Stock Center.
Endogenous gene replacements were generated using a scarless CRISPR strategy (http://flycrispr.molbio.wisc.edu/scarless). In brief, donor and gRNA plasmids were co-injected into vas-Cas 9 expressing Drosophila embryos (either BDRC 56552 or 51324 stocks). The donor plasmid contained the transgene of interest, $\sim 1 \mathrm{~kb}$ of homologous sequence upstream and downstream of the insertion sites, and a dsRed cassette as a selection marker. Additionally, the PAM sites that were used to guide Cas 9 cleavage of the endogenous genome were mutated in the donor plasmid to prevent cleavage of the transgene after insertion. The gRNA plasmid contained two gRNA sequences to direct Cas9 cleavage of sites upstream and downstream of the target genomic region to be replaced. The injection services of Bestgene were used to generate transgenic flies. Transgenic animals were verified by PCR and sequencing and the dsRed cassette was removed by crossing flies to $3 \times \mathrm{PP} 3$ transposase expressing flies. See Supplementary Table 2 for a list of all primers used in this study.

In vitro binding assays. $\mathrm{MBP}$ and GST fusion proteins were affinity purified in column buffer ( $20 \mathrm{mM}$ Tris (pH 7.0), $150 \mathrm{mM} \mathrm{NaCl}, 2 \mathrm{mM}$ EDTA, and $0.1 \%$ NP40) with Amylose Resin (NEB) or GST-bind resin (Millipore), respectively. $\mathrm{MBP}$ fusion proteins were eluted with maltose and GST fusion proteins were eluted with Glutathione per manufacturers recommendations. For binding reactions, $1 \mu \mathrm{g}$ of each protein was incubated with $50 \mu$ GST-bind resins in NP40 buffer for $1 \mathrm{hr}$ Beads were washed $3 \times$ with NP40 buffer before $50 \mu$ of $5 \times$ Sample Buffer was added. Proteins were boiled for $10 \mathrm{~min}$ and resolved by SDS-PAGE on a $4-12 \%$ Bis-Tris gel (ThermoFisher). Protein gels were stained with Coomassie Blue (Biorad) for $30 \mathrm{~min}$ and destained for $2-3 \mathrm{~h}$ in destain buffer $(40 \% \mathrm{MeOH}$ and $10 \%$ Acetic Acid) before imaging. For in vivo pull-down assays, purified recombinant proteins were left on beads and incubated with protein lysates in NP40 buffer for 2 h. Beads were washed $3 \times$ with NP40 buffer before adding $50 \mu \mathrm{l}$ of $5 \times$ sample buffer. Proteins were separated by SDS-PAGE and immunoblotted with anti-VCP (Cell Signaling, 7F3, Rabbit mAb \#2649) and anti-GFP (mouse monoclonal, Invitrogen clone 3E6; A-11120). Total lysates were immunoblotted with anti-tubulin (E7-c, DSHB). HRP conjugated goat anti-mouse and goat anti-rabbit secondary antibodies (ThermoFisher) were used at 1:2000.

Microscopy methods. For live imaging, 3rd instar larvae or adult fly abdomens were dissected in HL3 saline buffer with no calcium $(70 \mathrm{mM} \mathrm{NaCl}, 5 \mathrm{mM} \mathrm{KCl}, 10$ $\mathrm{mM} \mathrm{MgCl}_{2}, 10 \mathrm{mM} \mathrm{NaHCO}_{3}, 115 \mathrm{mM}$ sucrose, $4.2 \mathrm{mM}$ trehalose, and $5 \mathrm{mM}$ HEPES) and all live imaging was performed in saline. Imaging was performed on an inverted Axiovert 200 microscope (Zeiss) using a 100× Plan Apochromat objective (1.4NA). Images were captured with a CoolSnap HQ2 CCD camera (Photometrics) and de-convolved using Slidebook 5.0 software (Intelligent imaging innovations, Denver, CO). Image quantification was performed with ImageJ software $(\mathrm{NIH})$. Volume rendering was performed with Slidebook 5.0 software. Any adjustment of brightness or contrast was performed using Slidebook 5.0 software and always applied to the entire image.

For fixed imaging, adult brains were dissected in O'dowd's saline buffer (101 $\mathrm{mM} \mathrm{NaCl}, 1 \mathrm{mM} \mathrm{CaCl} 2,4 \mathrm{mM} \mathrm{MgCl}_{2}, 3 \mathrm{mM} \mathrm{KCl}, 5 \mathrm{mM}$ glucose, $1.25 \mathrm{mM}$ $\mathrm{NaH}_{2} \mathrm{PO}_{4}$, and $20.7 \mathrm{mM} \mathrm{NaHCO} 3, \mathrm{pH} \mathrm{7.247}$ ) and fixed in 4\% PFA for at least $1 \mathrm{~h}$. Adult abdominal muscles, DLMs, and TTMs were fixed in 4\% PFA for $1 \mathrm{~h}$. Muscles were stained with Phalloidin (ThermoFisher) overnight in a final concentration of $0.165 \mu \mathrm{M}$. Adult NMJs were visualized using mouse anti-BRP (1:100; Developmental Studies Hybridoma Bank) and rabbit anti-DLG $\left(1: 1000^{48}\right)$. Conjugated secondary antibodies (Alexa Fluor 488 goat anti-mouse and Cy3 goat anti-rabbit) were used at 1:300 (Jackson immuno-research laboratories). The DLMs and TTMs were dissected out of the thorax and identified by three parameters: size, shape, and location in the thorax. For co-localization analyses, Mander's correlation coefficients were calculated using the Coloc2 plugin in FIJI.

ApopTag staining. The central nervous system was dissected out of 30-day-old adult Drosophila, across indicated genotypes. All fat bodies and trachea were removed from the nervous tissue because it is auto-fluorescent. Tissue was adhered to a glass coverslip with Vectabond (Vector labs) and fixed in 1\% PFA overnight at $4{ }^{\circ} \mathrm{C}$. $1 \%$ PFA in PBS was prepared fresh, as premixed PFA in a methanol stabilizer disrupts ApopTag assays. Post-fix processing was performed in Ethanol:Acetic acid (2:1) for $10 \mathrm{~min}$ at $-20^{\circ} \mathrm{C}$. ApopTag assay was performed as described in Millipore Sigma ApopTag Fluorescein kit protocol. Tissue was mounted in vectasheild mounting media (Vector Labs) containing DAPI as a counterstain to image nuclei in the sample. ApopTag Fluorescein signal was imaged in the FITC channel. Samples were imaged on a Nikon Ti-E spinning disc confocal microscope with an Andor Zyla 5.2 camera, using micromanager software. Images were captured with a Plan Apo $\lambda \times 20 / 0.75$ Nikon objective. ApopTag Fluorescein positive nuclei were counted using FIJI 3D Object counter.

EM methods. Animals were fixed with $2 \%$ glutaraldehyde in $0.1 \mathrm{M}$ Nacacodylate buffer, $\mathrm{pH} 7.3$, for $2 \mathrm{~h}$ at room temp, rinsed in buffer, and post-fixed with $1 \% \mathrm{OsO}_{4}$ in a buffer for $1 \mathrm{~h}$ at room temp. The post-fixed samples were then rinsed with water, stained en bloc with $5 \%$ uranyl acetate in water, dehydrated in ethanol and propylene oxide, and embedded in Eponate 12 resin (Ted Pella, Inc., Redding, CA). $50 \mathrm{~nm}$ sections were cut with a Leica UCT ultramicrotome using a Diatome 
diamond knife, picked up on slot grids with Pioloform films, stained with uranyl acetate and Sato's lead, and examined with an FEI T12 TEM at $120 \mathrm{kV}$ equipped with a Gatan Ultrascan $4 \mathrm{k} \times 4 \mathrm{k}$ camera.

Lifespan and climbing assays. Adult male flies were collected within $12 \mathrm{~h}$ of eclosion and maintained at $25^{\circ} \mathrm{C}$ on standard fly food. Flies were flipped to new food and scored for death every 2-3 days. Climbing assays were performed by flipping flies into a $100 \mathrm{ml}$ graduated cylinder, tapping them to the bottom, and scoring the number of flies that could successfully climb to the $100 \mathrm{ml}$ mark in 1 $\mathrm{min}$. The statistical software, OASIS ${ }^{49}$, was used to calculate the mean lifespans and perform log-rank tests to determine statistical significance.

Electrophysiology methods. Adult Drosophila was immobilized in dental wax, ventral side down and a glass microelectrode was directly placed into the identified muscle through the cuticle of the animal ${ }^{37}$. Glass electrodes were filled with $3 \mathrm{M}$ $\mathrm{KCl}(40-60 \mathrm{M} \Omega$ resistance) and signals were amplified with an Axopatch $200 \mathrm{~B}$ (Axon Instruments). Giant fibers were stimulated extracellularly with a Master 8 stimulator through tungsten wire electrodes placed in each of the Drosophila eyes. An Axon Instruments Digidata 1322 A Data Acquisition System was used to digitize the data and recordings were collected with Clampex 10.7 software (Molecular Devices). Muscle depolarization at low $(0.2 \mathrm{~Hz})$ and high stimulus frequencies $(100 \mathrm{~Hz})$ were recorded to test the fidelity of the circuit in control and experimental animals.

Sequencing data generation and processing. Whole-genome sequencing of the patient's genomic DNA isolated from whole blood was performed as described previously ${ }^{50}$ at HudsonAlpha Institute for Biotechnology (Huntsville, AL) on an Illumina HiSeq-X, with 150 bp paired-end reads to obtain $30 \times$ sequencing coverage. Paired-end reads were aligned to the GRCh37 build of the human reference genome with the Burrows-Wheeler Aligner (BWA-MEM v0.7.8) and processed using the Broad Institute's Genome Analysis Toolkit (GATK) best-practices pipeline. The variant was confirmed with direct Sanger sequencing at UCSF.

Statistical methods. All Student's $t$-test were performed with two-tailed analysis. All ANOVA are one-way with Tukey's multiple comparisons. Lifespan data were compared using a log-rank test (see Lifespan, above). Analyses performed with Prism v9 (Graphpad).

Reporting summary. Further information on research design is available in the Nature Research Reporting Summary linked to this article.

\section{Data availability}

Further information and requests for resources and reagents should be directed to and will be fulfilled by the corresponding author Graeme Davis (Graeme.Davis@ucsf.edu). All unique/stable reagents generated in this study are available without restriction. This study did not generate data sets for public repositories or new data code. Source Data are provided with this paper.

Received: 18 February 2020; Accepted: 17 December 2020;

Published online: 21 January 2021

\section{References}

1. Baek, G. H. et al. Cdc48: a swiss army knife of cell biology. J. Amino Acids 2013, 183421 (2013).

2. Watts, G. D. J. et al. Inclusion body myopathy associated with Paget disease of bone and frontotemporal dementia is caused by mutant valosin-containing protein. Nat. Genet. 36, 377-381 (2004).

3. Abramzon, Y. et al. Valosin-containing protein (VCP) mutations in sporadic amyotrophic lateral sclerosis. Neurobiol. Aging 33, 2231.e1-2231.e6 (2012).

4. Tang, W. K. \& Xia, D. Mutations in the human AAA + chaperone p97 and related diseases. Front. Mol. Biosci. 3, 79 (2016).

5. Clemen, C. S. et al. Strumpellin is a novel valosin-containing protein binding partner linking hereditary spastic paraplegia to protein aggregation diseases. Brain 133, 2920-2941 (2010).

6. Liewluck, T. et al. A novel VCP mutation underlies scapuloperoneal muscular dystrophy and dropped head syndrome featuring lobulated fibers. Muscle Nerve https://doi.org/10.1002/mus.24290 (2014).

7. Kim, N. C. et al. VCP is essential for mitochondrial quality control by PINK1/ Parkin and this function is impaired by VCP mutations. Neuron 78, 65-80 (2013).

8. Spina, S. et al. Phenotypic variability in three families with valosin-containing protein mutation. Eur. J. Neurol. 20, 251-258 (2013).
9. Kimonis, V. E. et al. Clinical studies in familial VCP myopathy associated with Paget disease of bone and frontotemporal dementia. Am. J. Med. Genet. A 146A, 745-757 (2008)

10. Weihl, C. C., Pestronk, A. \& Kimonis, V. E. Valosin-containing protein disease: inclusion body myopathy with Paget's disease of the bone and frontotemporal dementia. Neuromuscul. Disord. 19, 308-315 (2009).

11. Moir, D., Stewart, S. E., Osmond, B. C. \& Botstein, D. Cold-sensitive celldivision-cycle mutants of yeast: isolation, properties, and pseudoreversion studies. Genetics 100, 547-563 (1982).

12. Leon, A. \& McKearin, D. Identification of TER94, an AAA ATPase protein, as a bam-dependent component of the Drosophila fusome. Mol. Biol. Cell 10, 3825-3834 (1999).

13. Chang, Y.-C. et al. Pathogenic VCP/TER94 alleles are dominant actives and contribute to neurodegeneration by altering cellular ATP level in a Drosophila IBMPFD model. PLoS Genet. 7, e1001288 (2011).

14. Tresse, E. et al. VCP/p97 is essential for maturation of ubiquitin-containing autophagosomes and this function is impaired by mutations that cause IBMPFD. Autophagy 6, 217-227 (2010).

15. Ju, J.-S. et al. Valosin-containing protein (VCP) is required for autophagy and is disrupted in VCP disease. J. Cell Biol. 187, 875-888 (2009).

16. Johnson, A. E., Shu, H., Hauswirth, A. G., Tong, A. \& Davis, G. W. VCPdependent muscle degeneration is linked to defects in a dynamic tubular lysosomal network in vivo. elife 4, e07366 (2015).

17. Nalbandian, A. et al. The multiple faces of valosin-containing proteinassociated diseases: inclusion body myopathy with Paget's disease of bone, frontotemporal dementia, and amyotrophic lateral sclerosis. J. Mol. Neurosci. 45, 522-531 (2011)

18. Halawani, D. \& Latterich, M. p97: The cell's molecular purgatory? Mol. Cell 22, 713-717 (2006).

19. Meyer, H., Bug, M. \& Bremer, S. Emerging functions of the VCP/p97 AAAATPase in the ubiquitin system. Nat. Cell Biol. 14, 117-123 (2012).

20. Rabinovich, E., Kerem, A., Fröhlich, K.-U., Diamant, N. \& Bar-Nun, S. AAAATPase p97/Cdc48p, a cytosolic chaperone required for endoplasmic reticulum-associated protein degradation. Mol. Cell. Biol. 22, 626-634 (2002).

21. Bento, A. C. et al. UBXD1 is a mitochondrial recruitment factor for $\mathrm{p} 97 / \mathrm{VCP}$ and promotes mitophagy. Sci. Rep. 8, 12415 (2018).

22. Zhang, T., Mishra, P., Hay, B. A., Chan, D. \& Guo, M. Valosin-containing protein (VCP/p97) inhibitors relieve Mitofusin-dependent mitochondrial defects due to VCP disease mutants. elife 6, e17834 (2017).

23. Custer, S. K., Neumann, M., Lu, H., Wright, A. C. \& Taylor, J. P. Transgenic mice expressing mutant forms $\mathrm{VCP} / \mathrm{p} 97$ recapitulate the full spectrum of IBMPFD including degeneration in muscle, brain and bone. Hum. Mol. Genet. 19, 1741-1755 (2010).

24. Ballar, P. et al. Identification of SVIP as an endogenous inhibitor of endoplasmic reticulum-associated degradation. J. Biol. Chem. 282, 33908-33914 (2007).

25. Nagahama, M. et al. SVIP is a novel VCP/p97-interacting protein whose expression causes cell vacuolation. Mol. Biol. Cell 14, 262-273 (2003).

26. Wang, Y. et al. SVIP induces localization of p97/VCP to the plasma and lysosomal membranes and regulates autophagy. PLoS ONE 6, e24478 (2011).

27. Hänzelmann, P. \& Schindelin, H. The structural and functional basis of the p97/valosin-containing protein (VCP)-interacting motif (VIM): mutually exclusive binding of cofactors to the N-terminal domain of p97. J. Biol. Chem. 286, 38679-38690 (2011)

28. Sweeney, S. T. \& Davis, G. W. Unrestricted synaptic growth in spinster-a late endosomal protein implicated in TGF-beta-mediated synaptic growth regulation. Neuron 36, 403-416 (2002).

29. Mauvezin, C., Ayala, C., Braden, C. R., Kim, J. \& Neufeld, T. P. Assays to monitor autophagy in Drosophila. Methods 68, 134-139 (2014)

30. Fouillet, A. et al. ER stress inhibits neuronal death by promoting autophagy. Autophagy 8, 915-926 (2012).

31. Chow, C. Y., Wolfner, M. F. \& Clark, A. G. Using natural variation in Drosophila to discover previously unknown endoplasmic reticulum stress genes. Proc. Natl Acad. Sci. USA 110, 9013-9018 (2013).

32. Razzaq, A. et al. Amphiphysin is necessary for organization of the excitationcontraction coupling machinery of muscles, but not for synaptic vesicle endocytosis in Drosophila. Genes Dev. 15, 2967-2979 (2001).

33. Pielage, J., Fetter, R. D. \& Davis, G. W. Presynaptic Spectrin is essential for synapse stabilization. Curr. Biol. 15, 918-928 (2005).

34. Eaton, B. A., Fetter, R. D. \& Davis, G. W. Dynactin is necessary for synapse stabilization. Neuron 34, 729-741 (2002).

35. Cataldo, A. M., Hamilton, D. J., Barnett, J. L., Paskevich, P. A. \& Nixon, R. A. Properties of the endosomal-lysosomal system in the human central nervous system: disturbances mark most neurons in populations at risk to degenerate in Alzheimer's disease. J. Neurosci. 16, 186-199 (1996).

36. Yin, H. Z. et al. Slow development of ALS-like spinal cord pathology in mutant valosin-containing protein gene knock-in mice. Cell Death Dis. 3 , e374-e374 (2012). 
37. Orr, B. O., Borgen, M. A., Caruccio, P. M. \& Murphey, R. K. Netrin and Frazzled regulate presynaptic gap junctions at a Drosophila giant synapse. J. Neurosci. 34, 5416-5430 (2014).

38. Allen, M. J., Godenschwege, T. A., Tanouye, M. A. \& Phelan, P. Making an escape: development and function of the Drosophila giant fibre system. Semin. Cell Dev. Biol. 17, 31-41 (2006).

39. van Oosten-Hawle, P., Porter, R. S. \& Morimoto, R. I. Regulation of organismal proteostasis by transcellular chaperone signaling. Cell 153, 1366-1378 (2013).

40. Demontis, F. \& Perrimon, N. FOXO/4E-BP signaling in Drosophila muscles regulates organism-wide proteostasis during aging. Cell 143, 813-825 (2010).

41. Fernando, R., Drescher, C., Nowotny, K., Grune, T. \& Castro, J. P. Impaired proteostasis during skeletal muscle aging. Free Radic. Biol. Med. 132, 58-66 (2019).

42. Kaushik, S. \& Cuervo, A. M. Proteostasis and aging. Nat. Med. 21, 1406-1415 (2015).

43. Klaips, C. L., Jayaraj, G. G. \& Hartl, F. U. Pathways of cellular proteostasis in aging and disease. J. Cell Biol. 217, 51-63 (2018).

44. Rubinsztein, D. C. The roles of intracellular protein-degradation pathways in neurodegeneration. Nature 443, 780-786 (2006).

45. Yerbury, J. J. et al. Walking the tightrope: proteostasis and neurodegenerative disease. J. Neurochem. 137, 489-505 (2016).

46. Rubinsztein, D. C., Mariño, G. \& Kroemer, G. Autophagy and aging. Cell 146, 682-695 (2011).

47. Gu, H. \& O’Dowd, D. K. Cholinergic synaptic transmission in adult Drosophila Kenyon cells in situ. J. Neurosci. 26, 265-272 (2006).

48. Budnik, V. et al. Regulation of synapse structure and function by the Drosophila tumor suppressor gene dlg. Neuron 17, 627-640 (1996).

49. Han, S. K. et al. OASIS 2: online application for survival analysis 2 with features for the analysis of maximal lifespan and healthspan in aging research. Oncotarget 7, 56147-56152 (2016).

50. Geier, E. G. et al. Rare variants in the neuronal ceroid lipofuscinosis gene MFSD8 are candidate risk factors for frontotemporal dementia. Acta Neuropathol. 137, 71-88 (2018).

\section{Acknowledgements}

This study was funded by NINDS grant R35NS097212 (G.W.D.); NINDS grant K99/ R00NS100988 (A.E.J.); NIH-NIA K01 AG049152 (J.S.Y.); NIH-NIA R01 AG062588 (J.S.Y.); Rainwater Charitable Foundation (J.S.Y.); NIA P01 AG019724 (B.L.M.); NIA P50 AG023501 (B.L.M.); and NIA P30 AG062422 (B.L.M.).

\section{Author contributions}

A.E.J. designed and conducted experiments including imaging, biochemistry, genetics, transgene and CRISPR transgenics, lifespan analyses, wrote, and edited the manuscript. B.O.O. contributed anatomical analysis of synapse and dendrite degeneration, cell death, and electrophysiology, and edited manuscript. R.D.F. contributed to electron microscopy and analysis. L.A.P. contributed to image analysis. E.G.G., A.J.M., J.S.Y., and B.L.M. contributed to clinical data. G.W.D. designed experiments, coordinated experiments, secured funding, wrote, and edited manuscript.

\section{Competing interests}

The authors declare no competing interests.

\section{Additional information}

Supplementary information is available for this paper at https://doi.org/10.1038/s41467 020-20796-8.

Correspondence and requests for materials should be addressed to G.W.D.

Peer review information Nature Communications thanks the anonymous reviewers for their contributions to the peer review of this work. Peer review reports are available

Reprints and permission information is available at http://www.nature.com/reprints

Publisher's note Springer Nature remains neutral with regard to jurisdictional claims in published maps and institutional affiliations.

(i) Open Access This article is licensed under a Creative Commons Attribution 4.0 International License, which permits use, sharing, adaptation, distribution and reproduction in any medium or format, as long as you give appropriate credit to the original author(s) and the source, provide a link to the Creative Commons license, and indicate if changes were made. The images or other third party material in this article are included in the article's Creative Commons license, unless indicated otherwise in a credit line to the material. If material is not included in the article's Creative Commons license and your intended use is not permitted by statutory regulation or exceeds the permitted use, you will need to obtain permission directly from the copyright holder. To view a copy of this license, visit http://creativecommons.org/ licenses/by/4.0/.

(C) The Author(s) 2021 